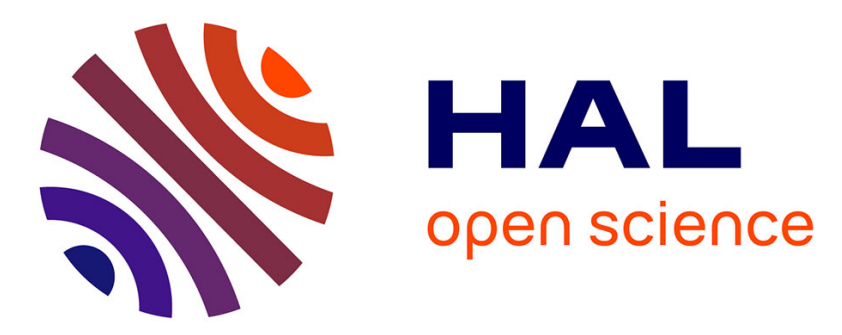

\title{
Analysis of the 1:1 resonant energy exchanges between coupled oscillators with rheologies
}

Alireza Ture Savadkoohi, Claude-Henri Lamarque, Mathieu Weiss, Bastien Vaurigaud, Simon Charlemagne

\section{- To cite this version:}

Alireza Ture Savadkoohi, Claude-Henri Lamarque, Mathieu Weiss, Bastien Vaurigaud, Simon Charlemagne. Analysis of the 1:1 resonant energy exchanges between coupled oscillators with rheologies. Nonlinear Dynamics, 2016, 86 (4), pp.2145-2159. 10.1007/s11071-016-2792-3 . hal-01308189

\author{
HAL Id: hal-01308189 \\ https://hal.science/hal-01308189
}

Submitted on 27 May 2020

HAL is a multi-disciplinary open access archive for the deposit and dissemination of scientific research documents, whether they are published or not. The documents may come from teaching and research institutions in France or abroad, or from public or private research centers.
L'archive ouverte pluridisciplinaire HAL, est destinée au dépôt et à la diffusion de documents scientifiques de niveau recherche, publiés ou non, émanant des établissements d'enseignement et de recherche français ou étrangers, des laboratoires publics ou privés. 


\title{
Analysis of the 1: 1 resonant energy exchanges between coupled oscillators with rheologies
}

\author{
A. Ture Savadkoohi · C.-H. Lamarque • \\ M. Weiss · B. Vaurigaud · S. Charlemagne
}

Received: date / Accepted: date

\begin{abstract}
The paper is composed of three main parts: the first part presents a two degrees of freedom coupled oscillators with rheology. One of the oscillators is intended to be the main structure and the second one is a nonlinear energy sink. The rheology of the system is represented via a set of internal variables that are governed by either differential inclusions or differential equations or direct algebraic relations between system variables. A step by step methodology is explained to trace system behaviors around a 1:1 resonance at different time scales. Invariant of the system at fast time scale is detected while possible periodic and strongly modulated regimes around its invariant are traced at slow time scales. The second part of the paper considers a set of several degrees of freedom main oscillators which are coupled to several nonlinear energy sinks. The overall system can house several rheologies. Explained methodology of the first part is expanded to this general case for tracing system responses at different time scales around 1:1 resonances. The third part of the paper presents two practical examples: The proposed methodology is used to detect invariants of systems and their equilibrium and singular points. This methodology provides some tools for designing equilibrium and singular points, i.e. periodic and strongly modulated regimes which lead to the design of nonlinear energy sinks for passively controlling and/or energy harvesting of the main oscillators.
\end{abstract}

Keywords energy transfer, $1: 1$ resonance, nonlinear energy sink, time multi scale behavior, passive control, energy harvesting, rheology

A. Ture Savadkoohi, C.-H. Lamarque, M. Weiss, S. Charlemagne Univ Lyon, ENTPE, LTDS UMR CNRS 5513, rue Maurice Audin, F-69518 Vaulx-en-Velin Cedex, France

Tel.: $+33(0) 472047246$

Fax: +33(0)4 72047156

E-mail: alireza.turesavadkoohi@entpe.fr

B. Vaurigaud

Cerema, Direction territoriale Sud-Ouest DLB, Bordeaux, France 


\section{Introduction}

Energy exchanges between two oscillators can be tuned for passive control or harvesting the energy of one of them. One of the well known methods for this aim is coupling a linear oscillator as the controller or the harvester to main systems. This linear oscillator is known as Frahm or tuned mass damper [1]. This system is quite heavy and modifies the frequency of the main structural systems while is efficient for narrow frequency ranges. In the past decade the nonlinear energy sink (NES) device is proposed for passive control or for energy harvesting of main structural systems [2-15]. Most of past studies consider that the NES possesses cubic nonlinearity [15] and the main structure is linear or presents smooth nonlinearity [16]. Some studies have been carried out which consider other types of nonlinearities for the NES such as vibro-impact and non-polynomial nonlinearities [17-21] or non-smooth potential function with constant or time-dependent mass [22-25]. Meanwhile, some research works have been studied which consider nonsmooth main oscillator and coupled nonsmooth or cubic NES. For instance, following coupled oscillators have been studied: a main oscillator with piecewise linear, Dahl-type and hardening elasto-plastic behaviors which are coupled nonsmooth NES [26-28]; a main oscillator with hysteresis behavior of Bouc-Wen type and a NES with general nonlinear potential function [29]; a main structure with single or several Saint-Venant elements [30] in parallel and a NES with cubic or general potential functions $[31,32]$. In this paper, we present a general methodology for studying the time multi-scale responses of main oscillators with general behaviors (nonlinear, nosmooth, ...) and coupled NES with general potentials (smooth, nonsmooth,...). The paper is followed by two special cases which consider two different types of coupled systems with different rheologies. Organization of the paper is as it follows: The general academic model of a two degrees of freedom (dof) system is presented in Sect.2. Primary treatments of system equations, i.e. possible change of system coordinates, complexification and using a Galerkin method are described in Sect. 2.1. System behaviors at fast and slow time scales which contains tracing invariant manifold of the system, its equilibrium and singular points are explained in Sect. 2.2. Another general system with rheologies composed of several dof main forced oscillators and several coupled NES is studied in Sect. 3. There, explained methodology in previous section is expanded to detect invariant, equilibrium points and singularities of considered several dof coupled oscillators. In Sect. 4 explained general methodology is endowed to study time multi-scale energy exchanges between a main linear system and a nonsmooth NES in the gravitational field. Sect. 5 uses the same methods for detection of time multiscale behaviors of a main system with hysteresis behavior which is coupled to a NES with cubic potential function. Finally, the paper is concluded in Sect. 6 .

\section{A two dof system with internal variables in the main dof}

The system under consideration is composed of two coupled oscillators: The main one with the mass 1 , the displacement $x$ and internal variables $z_{j}$ and $\dot{z}_{j}(j=1, \ldots, n)$ (representing parameters of a rheology) is under external force $\epsilon f_{0} \sin (\omega T)$. This oscillator is attached to a NES with the displacement $y$, the mass $\epsilon$ and a cubic or nonsmooth 
potential function. We summarize governing equations of the system as:

$$
\left\{\begin{array}{l}
\underbrace{\ddot{x}+\tilde{f}_{1}\left(x, \dot{x}, z_{1}, \ldots, z_{n}, \dot{z}_{1}, \ldots, \dot{z}_{n}\right)}_{\text {initial system with internal variables } z_{j}, \dot{z}_{j}, j=1, \ldots, n}+\underbrace{\tilde{f}_{2}(x, \dot{x}, y, \dot{y}}_{\text {coupling }})=\epsilon f_{0} \sin (\omega T) \\
\underbrace{\epsilon \ddot{y}-\epsilon f_{2}(y, \dot{y}, x, \dot{x})}_{\text {additional dof }(\mathrm{NES})}=0 \\
\begin{array}{l}
\text { Rheology } \rightarrow z_{1}, \ldots, z_{n}, \dot{z}_{1}, \ldots, \dot{z}_{n}(\text { Dimension } n) . \text { Constitutive laws for } z_{1}, \ldots, z_{n} \\
\text { in neighbourhood of }(0, \ldots, 0) .
\end{array}
\end{array}\right.
$$

Internal variables $z_{j}$ and $\dot{z}_{j}(j=1, \ldots, n)$ are governed by either differential inclusions, eg. $S^{t}$-Venant elements [30], or differential equations, eg. Bouc-Wen models [35], or piece-wise direct algebraic relations between $z_{j}, \dot{z}_{j}, x$ and $\dot{x}$ (or $y$ and $\dot{y}$ ) via $\tilde{f}_{1}\left(x, \dot{x}, z_{1}, \ldots, z_{n}, \dot{z}_{1}, \ldots, \dot{z}_{n}\right)$.

We emphasis that:

- Equations 1 are re-scaled forms of original system equations with respect to the new time domain $T$.

- The parameter $\epsilon$ which is in fact the mass ratio of the main oscillator and the NES, is very small: $0<\epsilon \ll 1$.

- We study system behavior around a $1: 1$ resonance. This is carried out by setting $\omega=\omega_{0}+\sigma \epsilon$, where $\omega_{0}$ is the re-scaled linear frequency of the main system, eg. $\omega_{0}=1$. The detuning parameter $\sigma$ permits to detect system behaviors around the $1: 1$ resonance.

In order to study and to predict behaviors of two oscillators at different scales of time, the system 1 is treated as it follows:

2.1 Possible change of system coordinates, complexifications and using the Galerkin technique

Some preliminary treatments of system 1 are carried out in order to prepare it for the time multi-scale analysis. They are listed here:

- Linear transformation for $x$ and $y$ coordinates via $\mathbb{A}$ matrix:

$$
\left(\begin{array}{c}
v \\
w
\end{array}\right)=\mathbb{A}\left(\begin{array}{l}
x \\
y
\end{array}\right)
$$

For instance, transferring the system to the center of the mass and the relative displacement of two oscillators:

$$
\mathbb{A}=\left(\begin{array}{cc}
1 & \epsilon \\
1 & -1
\end{array}\right)
$$

- Depending on the condition of the system, complex variables of Manevitch (for systems without pre-stress) [33] or their extended versions (for pre-stressed systems) 
$[23,28]$ are applied $\left(i^{2}=-1\right)$ :

$$
\left\{\begin{array}{l}
i b_{1}+\varphi_{1} e^{i \omega T}=\dot{v}+i \omega v \\
i b_{2}+\varphi_{2} e^{i \omega T}=\dot{w}+i \omega w \\
\varphi_{3 j} e^{i \omega T}=\dot{z}_{j}+i \omega z_{j} \quad j=1, \ldots, n
\end{array}\right.
$$

It should be mentioned that we can consider higher harmonics such as $\left(\varphi_{12} e^{2 i \omega T}+\right.$ $\left.\varphi_{13} e^{3 i \omega T}+\ldots\right),\left(\varphi_{22} e^{2 i \omega T}+\varphi_{23} e^{3 i \omega T}+\ldots\right)$ and $\left(\varphi_{3 j 2} e^{2 i \omega T}+\varphi_{3 j 3} e^{3 i \omega T}+\ldots\right)$.

- We consider different scales of the time, fast time scale $\left(\tau_{0}\right)$, slow time scale $\left(\tau_{1}\right), \ldots$ connecting to each other by $\epsilon$ parameter:

$$
T=\tau_{0}, \quad \tau_{1}=\epsilon \tau_{0}, \quad \tau_{2}=\epsilon^{2} \tau_{0}, \quad \cdots
$$

- We use the Galerkin method, i.e. a truncated Fourier series (constant terms and first harmonics). For a general function $\urcorner\left(b_{1}, b_{2}, \varphi_{1}, \varphi_{2}, \varphi_{31}, \ldots, \varphi_{3 n}\right)$, it reads:

$$
\left.\chi\left(b_{1}, b_{2}, \varphi_{1}, \varphi_{2}, \varphi_{31}, \ldots, \varphi_{3 n}\right)=\frac{\omega}{2 \pi} \int_{0}^{\frac{2 \pi}{\omega}}\right\urcorner\left(b_{1}, b_{2}, \varphi_{1}, \varphi_{2}, \varphi_{31}, \ldots, \varphi_{3 n}\right) e^{-l i \omega T} d T
$$

with $l=0,1$ for evaluating constant terms and first harmonics of the Fourier series, respectively. For evaluating the integral of the Eq. 6, we assume that $b_{1}, b_{2}, \varphi_{1}$, $\varphi_{2}, \varphi_{31}, \ldots, \varphi_{3 n}$ do not depend on fast time scale, i.e. $\tau_{0}=T$. This will be either verified during the time multi-scale analysis of the system or we will suppose that after a long transient response, the system reaches to an asymptotic state which is independent to time $T$ (mathematically $\tau_{0} \rightarrow \infty$ ).

2.2 Detection of system behaviors at different scales of time

A multiple scale method [36] for detecting system behaviors at different scales of time is used. After embedding the time $T$ into different scales, i.e. via Eq. 5, we would like to study system behaviors at each time scale, which means that we should consider system equations at different orders of $\epsilon$. If we consider constant terms of Fourier series, i.e. by setting $l=0$ in Eq. 6 , then $\epsilon^{0}$ and $\epsilon^{1}$ orders of system equations provide both constant terms of Eq. $4, b_{1}$ and $b_{2}$, as a function of pre-stressing terms. In the next sections we will focus on the evolutions of first harmonics of the system at different time scales.

\subsubsection{System behavior at fast time scale: $\epsilon^{0}$ order of system equations}

Let us consider first harmonics of the system via setting $l=1$ in Eq. 6 . At fast time scale the system yields to:

$$
\begin{gathered}
\frac{\partial \varphi_{1}}{\partial \tau_{0}}=0 \Rightarrow \varphi_{1}=\varphi_{1}\left(\tau_{1}, \tau_{2}, \ldots\right) \\
\frac{\partial \varphi_{2}}{\partial \tau_{0}}+\Lambda\left(\varphi_{1}, \varphi_{2}, \varphi_{31}, \varphi_{32}, \ldots, \varphi_{3 n}\right)=0
\end{gathered}
$$




$$
\Upsilon\left(\varphi_{1}, \varphi_{2}, \varphi_{31}, \varphi_{32}, \ldots, \varphi_{3 n}\right)=0
$$

Let us consider Eq. 8 at the infinity of fast time scale, i.e. $\frac{\partial \varphi_{2}}{\partial \tau_{0}} \rightarrow 0$ :

$$
\Lambda\left(\varphi_{1}, \varphi_{2}, \varphi_{31}, \varphi_{32}, \ldots, \varphi_{3 n}\right)=0
$$

Equations 9 and 10 present an asymptotic equilibrium governed by a manifold called slow invariant manifold (SIM). Equations 7, 9 and 10 show that $\varphi_{1}, \varphi_{2}, \varphi_{31}, \ldots, \varphi_{3 n}$ are constant during the time $\tau_{0}=T$, so our assumption for evaluating the constant and first terms of Fourier series in Eq. 6 is verified a posteriori. We can distinguish two possible cases for the SIM:

\section{Case 1}

Let us assume that Eq. 9 provides following explicit relation:

$$
\varphi_{3 j}=\mathbb{H}_{3 j}\left(\varphi_{1}, \varphi_{2}\right), j=1, \ldots, n
$$

so, Eq. 10 reads:

$$
\Lambda\left(\varphi_{1}, \varphi_{2}, \varphi_{31}, \varphi_{32}, \ldots, \varphi_{3 n}\right)=0 \Leftrightarrow \tilde{\Lambda}\left(\varphi_{1}, \varphi_{2}\right)=0
$$

We set $\varphi_{j}=N_{j} e^{i \delta_{j}}, j=1,2$. There are two possibilities on the relation between $\varphi_{1}$ and $\varphi_{2}$ :

I There is an explicit relation between $\varphi_{1}$ and $\varphi_{2}$, i.e.:

$$
\varphi_{1}=\mathbb{H}\left(\varphi_{2}\right)
$$

or,

$$
\left\{\begin{array}{l}
\delta_{1}=h_{1}\left(\delta_{2}, N_{2}\right) \\
N_{1}=h_{2}\left(\delta_{2}, N_{2}\right)
\end{array} \Leftrightarrow\left(\begin{array}{c}
\delta_{1} \\
N_{1}
\end{array}\right)=h\left(\delta_{2}, N_{2}\right)\right.
$$

II There is not an explicit relation between $\varphi_{1}$ and $\varphi_{2}$. In this case Eq. 12 provides two relations:

$$
\tilde{\Lambda}\left(\varphi_{1}, \varphi_{2}\right)=0 \Leftrightarrow \widehat{\Lambda}\left(\delta_{2}, N_{2}, \delta_{1}, N_{1}\right)=\left(\begin{array}{l}
0 \\
0
\end{array}\right)
$$

Functions $\tilde{\Lambda}\left(\varphi_{1}, \varphi_{2}\right)$ are equations defined in the complex domain and $\widehat{\Lambda}\left(\delta_{2}, N_{2}, \delta_{1}, N_{1}\right)$ correspond to the same equations in the real domain.

\section{Case 2}

This case considers a very general form of the SIM supposing that there is not explicit relations between system variables. By combining Eqs. 9 and 10, the general form of the SIM can be written in the compact form as:

$$
\mathscr{S}\left(\varphi_{1}, \varphi_{2}, \varphi_{31}, \varphi_{32}, \ldots, \varphi_{3 n}\right)=0
$$

which is a set of equations in the complex domain. The same equations in the real domain read:

$$
\widehat{\mathscr{S}}\left(\delta_{2}, N_{2}, \delta_{1}, N_{1}, \delta_{31}, N_{31}, \ldots, N_{1}, \delta_{3 n}, N_{3 n}\right)=0
$$


2.2.2 System behavior at slow time scale: $\epsilon^{1}$ order of system equations

We are interested to study the system at slow time scale but around its SIM. This means that in the developments of system equations at $\epsilon^{1}$ order, we should consider equations of the SIM which are obtained in Sect.2.2.1. The core idea is to detect equilibrium and singular points of the system which correspond to its periodic regimes and strongly modulated responses (SMR) [34]. We can distinguish different scenarios according to different cases which have been defined in Sect.2.2.1:

\section{Scenario 1}

Let us suppose that the SIM follows assumptions of the Case 1:

I There is an explicit relation between $\varphi_{1}$ and $\varphi_{2}$.

$$
\frac{\partial \varphi_{1}}{\partial \tau_{1}}=\mathscr{H}\left(\varphi_{1}, \varphi_{2}\right) \Leftrightarrow\left(\begin{array}{c}
\frac{\partial \delta_{1}}{\partial \tau_{1}} \\
\frac{\partial N_{1}}{\partial \tau_{1}}
\end{array}\right)=\left(\begin{array}{c}
\mathscr{H}_{1}\left(\delta_{2}, N_{2}\right) \\
\mathscr{H}_{2}\left(\delta_{2}, N_{2}\right)
\end{array}\right)
$$

Let us consider evolution of the system along the SIM at $\tau_{1}$ time scale. We inject Eq. 14 in the Eq. 18,

$$
\nabla_{\left(\delta_{2}, N_{2}\right)} h\left(\begin{array}{c}
N_{2} \frac{\partial \delta_{2}}{\partial \tau_{1}} \\
\frac{\partial N_{2}}{\partial \tau_{1}}
\end{array}\right)=\widetilde{\mathscr{H}}\left(\delta_{2}, N_{2}\right)
$$

where $\nabla_{\left(\delta_{2}, N_{2}\right)} h$ denotes the jacobian matrix of $h$ versus variables $\delta_{2}$ and $N_{2}$ (similar notations are used here after). Equilibrium points of the system are those which provide:

$$
\left\{\begin{array}{c}
\widetilde{\mathscr{H}}\left(\delta_{2}, N_{2}\right)=0 \\
\& \\
\nabla_{\left(\delta_{2}, N_{2}\right)} h \text { be invertible }
\end{array}\right.
$$

while singular points provide:

$$
\left\{\begin{array}{c}
\widetilde{\mathscr{H}}\left(\delta_{2}, N_{2}\right)=0 \\
\& \\
\nabla_{\left(\delta_{2}, N_{2}\right)} h \text { not to be invertible }
\end{array}\right.
$$

II There is not an explicit relation between $\varphi_{1}$ and $\varphi_{2}$.

$$
\frac{\partial \varphi_{1}}{\partial \tau_{1}}=\mathscr{F}\left(\varphi_{1}, \varphi_{2}\right) \Leftrightarrow\left(\begin{array}{c}
\frac{\partial \delta_{1}}{\partial \tau_{1}} \\
\frac{\partial N_{1}}{\partial \tau_{1}}
\end{array}\right)=\left(\begin{array}{c}
\mathscr{F}_{1}\left(\delta_{2}, N_{2}, \delta_{1}, N_{1}\right) \\
\mathscr{F}_{2}\left(\delta_{2}, N_{2}, \delta_{1}, N_{1}\right)
\end{array}\right)
$$

Let us consider system modulations along the SIM at $\tau_{1}$ time scale. Equation 15 is derived versus $\tau_{1}$ time scale: 


$$
\nabla_{\left(\delta_{2}, N_{2}, \delta_{1}, N_{1}\right)} \widehat{\Lambda}\left(\begin{array}{c}
\frac{\partial \delta_{2}}{\partial \tau_{1}} \\
\frac{\partial N_{2}}{\partial \tau_{1}} \\
\frac{\partial \delta_{1}}{\partial \tau_{1}} \\
\frac{\partial N_{1}}{\partial \tau_{1}}
\end{array}\right)=\left(\begin{array}{l}
0 \\
0
\end{array}\right)
$$

The dimension of the matrix $\nabla \widehat{\Lambda}\left(\delta_{2}, N_{2}, \delta_{1}, N_{1}\right)$ is $(2 \times 4)$. In the blocked form it can be re-written as:

$$
\nabla_{\left(\delta_{2}, N_{2}, \delta_{1}, N_{1}\right)} \widehat{\Lambda}=\left[\nabla_{\left(\delta_{2}, N_{2}\right)} \widehat{\Lambda} \mid \nabla_{\left(\delta_{1}, N_{1}\right)} \widehat{\Lambda}\right]
$$

So, Eq. 23 reads:

$$
\nabla_{\left(\delta_{2}, N_{2}\right)} \widehat{\Lambda}\left(\begin{array}{c}
\frac{\partial \delta_{2}}{\partial \tau_{1}} \\
\frac{\partial N_{2}}{\partial \tau_{1}}
\end{array}\right)+\nabla_{\left(\delta_{1}, N_{1}\right)} \widehat{\Lambda}\left(\begin{array}{c}
\frac{\partial \delta_{1}}{\partial \tau_{1}} \\
\frac{\partial N_{1}}{\partial \tau_{1}}
\end{array}\right)=\left(\begin{array}{l}
0 \\
0
\end{array}\right)
$$

This equation can be rearranged by considering Eq. 22:

$$
\nabla_{\left(\delta_{2}, N_{2}\right)} \widehat{\Lambda}\left(\begin{array}{c}
\frac{\partial \delta_{2}}{\partial \tau_{1}} \\
\frac{\partial N_{2}}{\partial \tau_{1}}
\end{array}\right)=-\nabla_{\left(\delta_{1}, N_{1}\right)} \widehat{\Lambda}\left(\begin{array}{c}
\mathscr{F}_{1}\left(\delta_{2}, N_{2}, \delta_{1}, N_{1}\right) \\
\mathscr{F}_{2}\left(\delta_{2}, N_{2}, \delta_{1}, N_{1}\right)
\end{array}\right)
$$

Equations 15, 22 and 26 provide useful information relevant to positions of equilibrium and singular points. Equilibrium points of the system provide:

$$
\left\{\begin{array}{c}
\widehat{\Lambda}\left(\delta_{2}, N_{2}, \delta_{1}, N_{1}\right)=\left(\begin{array}{l}
0 \\
0
\end{array}\right) \\
\& \\
\mathscr{F}_{1}\left(\delta_{2}, N_{2}, \delta_{1}, N_{1}\right)=\mathscr{F}_{2}\left(\delta_{2}, N_{2}, \delta_{1}, N_{1}\right)=0 \\
\& \\
\nabla_{\left(\delta_{2}, N_{2}\right)} \widehat{\Lambda} \text { be invertible }
\end{array}\right.
$$

Singular points of the system provide:

$$
\left\{\begin{array}{c}
\widehat{\Lambda}\left(\delta_{2}, N_{2}, \delta_{1}, N_{1}\right)=\left(\begin{array}{l}
0 \\
0
\end{array}\right) \\
\& \\
\mathscr{F}_{1}\left(\delta_{2}, N_{2}, \delta_{1}, N_{1}\right)=\underset{F_{2}}{ }\left(\delta_{2}, N_{2}, \delta_{1}, N_{1}\right)=0 \\
\quad \& \\
\nabla_{\left(\delta_{2}, N_{2}\right)} \widehat{\Lambda} \text { not to be invertible }
\end{array}\right.
$$

\section{Scenario 2}

Let us consider a very general form of the SIM (see Eq. 16) which has been explained in Case 2 of the Sect. 2.2.1. 
$\epsilon^{1}$ order of system equations read (i.e. the system at slow time scale):

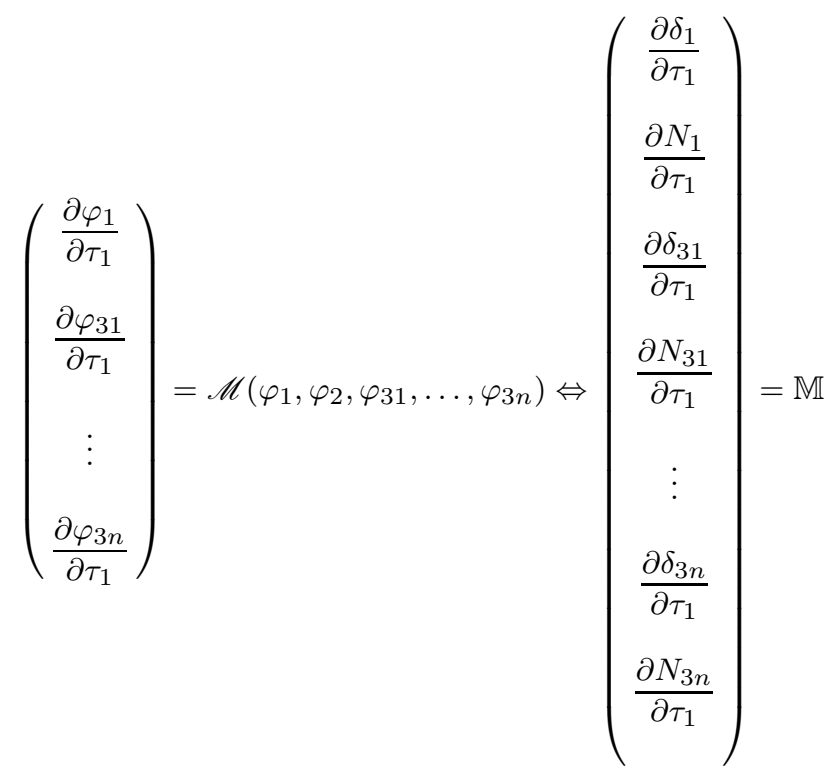

with

$$
\mathbb{M}=\left(\begin{array}{c}
\mathscr{M}_{1}\left(\delta_{2}, N_{2}, \delta_{1}, N_{1}, \delta_{31}, N_{31}, \ldots, N_{1}, \delta_{3 n}, N_{3 n}\right) \\
\mathscr{M}_{2}\left(\delta_{2}, N_{2}, \delta_{1}, N_{1}, \delta_{31}, N_{31}, \ldots, N_{1}, \delta_{3 n}, N_{3 n}\right) \\
\mathscr{M}_{3}\left(\delta_{2}, N_{2}, \delta_{1}, N_{1}, \delta_{31}, N_{31}, \ldots, N_{1}, \delta_{3 n}, N_{3 n}\right) \\
\vdots \\
\mathscr{M}_{2 n+3}\left(\delta_{2}, N_{2}, \delta_{1}, N_{1}, \delta_{31}, N_{31}, \ldots, N_{1}, \delta_{3 n}, N_{3 n}\right) \\
\mathscr{M}_{2 n+4}\left(\delta_{2}, N_{2}, \delta_{1}, N_{1}, \delta_{31}, N_{31}, \ldots, N_{1}, \delta_{3 n}, N_{3 n}\right)
\end{array}\right)
$$


Equation 17 can be derived versus $\tau_{1}$ time scale as:

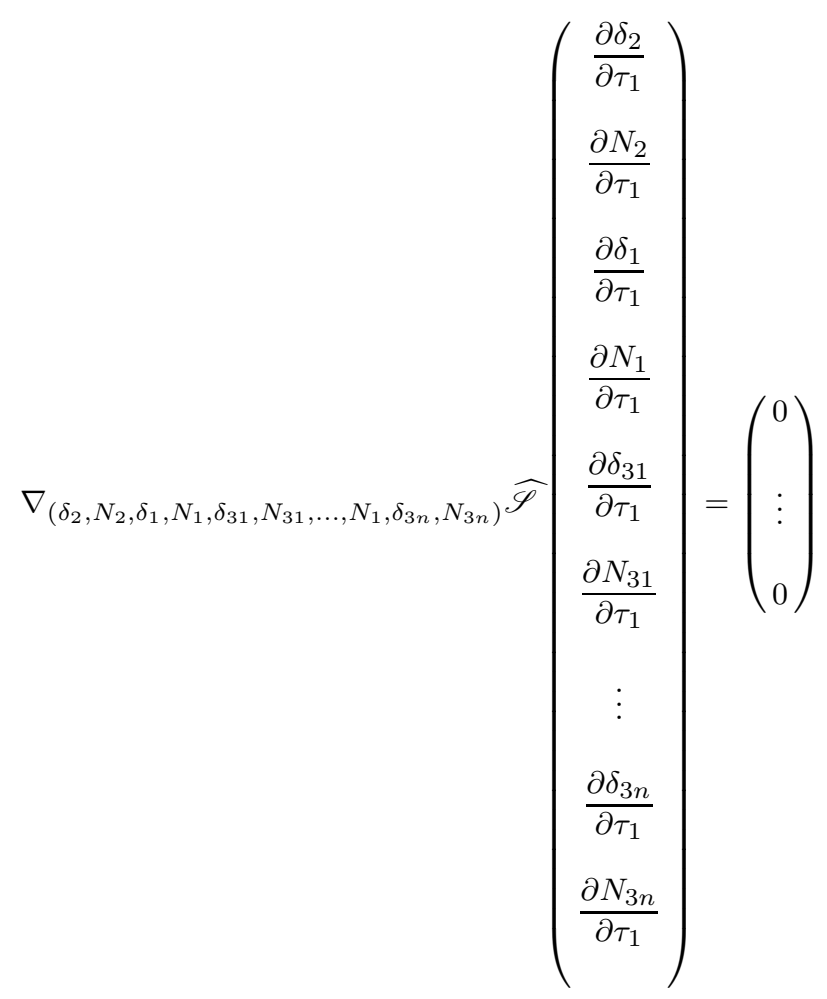

Dimension of the $\nabla_{\left(\delta_{2}, N_{2}, \delta_{1}, N_{1}, \delta_{31}, N_{31}, \ldots, N_{1}, \delta_{3 n}, N_{3 n}\right)} \widehat{\mathscr{S}}$ is $(2 n+2) \times(2 n+4)$. So, Eq. 31 provides $(2 n+2)$ relations. In the blocked form we can write:

$$
\begin{aligned}
& \nabla_{\left(\delta_{2}, N_{2}, \delta_{1}, N_{1}, \delta_{31}, N_{31}, \ldots, N_{1}, \delta_{3 n}, N_{3 n}\right)} \widehat{\mathscr{S}}= \\
& {\left[\nabla_{\left(\delta_{2}, N_{2}\right)} \widehat{\mathscr{S}} \mid \nabla_{\left(\delta_{1}, N_{1}, \delta_{31}, N_{31}, \ldots, N_{1}, \delta_{3 n}, N_{3 n}\right)} \widehat{\mathscr{S}}\right]}
\end{aligned}
$$


Equation 31 reads;

$$
\nabla_{\left(\delta_{2}, N_{2}\right)} \widehat{\mathscr{S}}\left(\begin{array}{c}
\frac{\partial \delta_{2}}{\partial \tau_{1}} \\
\frac{\partial N_{2}}{\partial \tau_{1}}
\end{array}\right)+\nabla_{\left(\delta_{1}, N_{1}, \delta_{31}, N_{31}, \ldots, N_{1}, \delta_{3 n}, N_{3 n}\right)} \widehat{\mathscr{S}}\left(\begin{array}{c}
\frac{\partial \delta_{1}}{\partial \tau_{1}} \\
\frac{\partial N_{1}}{\partial \tau_{1}} \\
\frac{\partial \delta_{31}}{\partial \tau_{1}} \\
\frac{\partial N_{31}}{\partial \tau_{1}} \\
\vdots \\
\frac{\partial \delta_{3 n}}{\partial \tau_{1}} \\
\frac{\partial N_{3 n}}{\partial \tau_{1}} \\
\vdots \\
0
\end{array}\right)=\left(\begin{array}{c}
0 \\
\vdots \\
\end{array}\right)
$$

or

$$
\nabla_{\left(\delta_{2}, N_{2}\right)} \widehat{\mathscr{S}}\left(\begin{array}{c}
\frac{\partial \delta_{2}}{\partial \tau_{1}} \\
\frac{\partial N_{2}}{\partial \tau_{1}}
\end{array}\right)=-\nabla_{\left(\delta_{1}, N_{1}, \delta_{31}, N_{31}, \ldots, N_{1}, \delta_{3 n}, N_{3 n}\right)} \widehat{\mathscr{S M}}
$$

Equilibrium and singular points of the system can be traced via Eqs. 17, 29 and 34. Equilibrium points can be detected via

$$
\left\{\begin{array}{c}
\widehat{\mathscr{S}}\left(\delta_{2}, N_{2}, \delta_{1}, N_{1}, \delta_{31}, N_{31}, \ldots, N_{1}, \delta_{3 n}, N_{3 n}\right)=0 \\
\& \\
\mathbb{M}=0 \\
\& \\
\nabla_{\left(\delta_{2}, N_{2}\right)} \widehat{\mathscr{S}} \text { be invertible }
\end{array}\right.
$$

Singular points of the system provide:

$$
\left\{\begin{array}{c}
\widehat{\mathscr{S}}\left(\delta_{2}, N_{2}, \delta_{1}, N_{1}, \delta_{31}, N_{31}, \ldots, N_{1}, \delta_{3 n}, N_{3 n}\right)=0 \\
\& \\
\mathbb{M}=0 \\
\& \\
\nabla_{\left(\delta_{2}, N_{2}\right)} \widehat{\mathscr{S}} \text { not to be invertible }
\end{array}\right.
$$

3 Time multi-scale energy exchanges between a multiple degrees of freedom main oscillator and multiple NES including rheologies

All developments which are explained in Sect.2 are based on a two dof system which includes a rheology of dimension $n$ (see Eq. 1). In this section we would like to expand 
all of previous developments to a system which is composed of $n_{m}$ dof main oscillators which are coupled to $n_{n}$ dof NES. The dimension of the rheology is assumed to be $n_{r}$. Let us assume that system equations read:

$$
\left\{\begin{array}{l}
\ddot{\boldsymbol{Y}}_{1}+\boldsymbol{\Delta}_{1} \boldsymbol{Y}_{1}+\epsilon \boldsymbol{\Delta}_{2} \dot{\boldsymbol{Y}}_{1}+\underbrace{\epsilon \mathfrak{F}_{1}\left(\boldsymbol{Y}_{1}, \boldsymbol{Y}_{2}\right)+\epsilon \mathfrak{F}_{2}\left(\dot{\boldsymbol{Y}}_{1}, \dot{\boldsymbol{Y}}_{2}\right)}_{\epsilon \tilde{\mathfrak{F}}\left(\boldsymbol{Y}_{1}, \boldsymbol{Y}_{2}, \dot{\boldsymbol{Y}}_{1}, \dot{\boldsymbol{Y}}_{2}\right)}+\mathfrak{E}\left(\boldsymbol{Y}_{1}, \boldsymbol{Y}_{2}, \dot{\boldsymbol{Y}}_{3}\right)=\epsilon \boldsymbol{f}_{0} \sin (\omega T) \\
\ddot{\boldsymbol{Y}}_{2}-\epsilon \tilde{\mathfrak{F}}\left(\boldsymbol{Y}_{1}, \boldsymbol{Y}_{2}, \dot{\boldsymbol{Y}}_{1}, \dot{\boldsymbol{Y}}_{2}\right)=\mathbf{0} \\
\dot{\boldsymbol{Y}}_{3}+\boldsymbol{G}\left(\boldsymbol{Y}_{1}, \boldsymbol{Y}_{2}, \boldsymbol{Y}_{3}\right)=\mathbf{0} \text { or }\left(\dot{\boldsymbol{Y}}_{3}+\boldsymbol{G}\left(\boldsymbol{Y}_{1}, \boldsymbol{Y}_{2}, \boldsymbol{Y}_{3}\right)\right) \ni \mathbf{0}
\end{array}\right.
$$

Where $\boldsymbol{Y}_{1}, \boldsymbol{Y}_{2}$ and $\boldsymbol{Y}_{3}$ are vectors of dimensions $n_{m}, n_{n}$ and $n_{r}$, respectively. $\boldsymbol{f}_{0}$ is the vector of applied external forcing amplitudes on each dof of the main system (with dimension $n_{m}$ ). Since we would like to study system behaviors around 1:1 resonances, we assume that:

$$
\boldsymbol{\Delta}_{1}=\omega^{2} \boldsymbol{I}_{d}+\epsilon \boldsymbol{\Sigma}
$$

$\boldsymbol{\Sigma}$ is a diagonal matrix of detuning parameters and $\boldsymbol{I}_{d}$ is the unity matrix. To detect system behaviors at different scales of time, we follow similar steps which have been explained in Sect.2.

3.1 Complexifications and using the Galerkin technique

System variables could be submitted to the following linear transformation:

$$
\left(\begin{array}{c}
\boldsymbol{V} \\
\boldsymbol{W}
\end{array}\right)=\mathbb{A}\left(\begin{array}{c}
\boldsymbol{Y}_{1} \\
\boldsymbol{Y}_{2}
\end{array}\right)
$$

In order to simplify, let us assume that $\mathbb{A}=\boldsymbol{I}_{d}$. Complex variables of Manevitch [33] or their extended versions $[23,28]$ are applied to system variables:

$$
\left\{\begin{array}{l}
i \boldsymbol{B}_{1}+\boldsymbol{\Phi}_{1} e^{i \omega T}=\dot{\boldsymbol{V}}+i \omega \boldsymbol{V} \\
i \boldsymbol{B}_{2}+\boldsymbol{\Phi}_{2} e^{i \omega T}=\dot{\boldsymbol{W}}+i \omega \boldsymbol{W} \\
\boldsymbol{\Phi}_{3} e^{i \omega T}=\dot{\boldsymbol{Y}}_{3}+i \omega \boldsymbol{Y}_{3}
\end{array}\right.
$$

The Galerkin technique is endowed for keeping constant and first harmonics of the Fourier series. For any arbitrary function $\boldsymbol{\top}\left(\boldsymbol{B}_{1}, \boldsymbol{B}_{1}, \boldsymbol{\Phi}_{1}, \boldsymbol{\Phi}_{2}, \boldsymbol{\Phi}_{3}\right)$ this task is carried out via:

$$
\left.\chi\left(\boldsymbol{B}_{1}, \boldsymbol{B}_{1}, \boldsymbol{\Phi}_{1}, \boldsymbol{\Phi}_{2}, \boldsymbol{\Phi}_{3}\right)=\frac{\omega}{2 \pi} \int_{0}^{\frac{2 \pi}{\omega}}\right\urcorner\left(\boldsymbol{B}_{1}, \boldsymbol{B}_{1}, \boldsymbol{\Phi}_{1}, \boldsymbol{\Phi}_{2}, \boldsymbol{\Phi}_{3}\right) e^{-l i \omega T} d T
$$

with $l=0,1$. For solving integrals of Eq. 41 we use the same assumptions that are explained in Sect. 2.1 . 
3.2 Time multi-scale behaviors of the system

If we use $l=0$ in Eq. 41, i.e. we keep constant terms of the Fourier series, then vectors $\boldsymbol{B}_{1}$ and $\boldsymbol{B}_{2}$ can be revealed by considering system equations at $\epsilon^{0}$ and $\epsilon^{1}$ orders. Let us concentrate at first harmonics of Fourier series, i.e. we set $l=1$ in Eq. 41. $\epsilon^{0}$ order of system equations provide:

$$
\begin{gathered}
\frac{\partial \boldsymbol{\Phi}_{1}}{\partial \tau_{0}}=0 \Rightarrow \boldsymbol{\Phi}_{1}=\boldsymbol{\Phi}_{1}\left(\tau_{1}, \tau_{2}, \ldots\right) \\
\frac{\partial \boldsymbol{\Phi}_{2}}{\partial \tau_{0}}+\boldsymbol{\Lambda}\left(\boldsymbol{\Phi}_{1}, \boldsymbol{\Phi}_{2}, \boldsymbol{\Phi}_{3}\right)=0 \\
\boldsymbol{\Upsilon}\left(\boldsymbol{\Phi}_{1}, \boldsymbol{\Phi}_{2}, \boldsymbol{\Phi}_{3}\right)=0
\end{gathered}
$$

Equation 42 provides $n_{m}$ relations in the complex domain. When $\tau_{0} \rightarrow \infty\left(\frac{\partial \boldsymbol{\Phi}_{2}}{\partial \tau_{0}}=0\right)$, Eq. 43 and 44 provide asymptotic responses of the system which are in fact its SIM. In the compact form and as a set of equations in the complex domain, it reads:

$$
\mathscr{S}\left(\boldsymbol{\Phi}_{1}, \boldsymbol{\Phi}_{2}, \boldsymbol{\Phi}_{3}\right)=\mathbf{0}
$$

which provides $n_{m}+n_{r}$ relations in the complex domain. Equations 45 in the real domain read:

$$
\widehat{\mathscr{S}}\left(\boldsymbol{\delta}_{2}, \boldsymbol{N}_{2}, \boldsymbol{\delta}_{1}, \boldsymbol{N}_{1}, \boldsymbol{\delta}_{3}, \boldsymbol{N}_{3}\right)=\mathbf{0}
$$

where $\boldsymbol{\delta}_{j}, \boldsymbol{N}_{j}, j=1,2,3$ are phase and amplitude vectors of $\boldsymbol{\Phi}_{j}, j=1,2,3$, respectively. Equation 46 provides $2\left(n_{m}+n_{r}\right)$ relations. Detection of system behaviors at slow time scale $\tau_{1}$ demands consideration of system equations at $\epsilon^{1}$ order. We can write:

$$
\left(\begin{array}{c}
\frac{\partial \boldsymbol{\Phi}_{1}}{\partial \tau_{1}} \\
\frac{\partial \boldsymbol{\Phi}_{3}}{\partial \tau_{1}}
\end{array}\right)=\mathscr{M}\left(\boldsymbol{\Phi}_{1}, \boldsymbol{\Phi}_{2}, \boldsymbol{\Phi}_{3}\right) \Leftrightarrow\left(\begin{array}{c}
\frac{\partial \boldsymbol{\delta}_{1}}{\partial \tau_{1}} \\
\frac{\partial \boldsymbol{N}_{1}}{\partial \tau_{1}} \\
\frac{\partial \boldsymbol{\delta}_{3}}{\partial \tau_{1}} \\
\frac{\partial \boldsymbol{N}_{3}}{\partial \tau_{1}}
\end{array}\right)=\mathbb{M}
$$


Let us find the evolution of the SIM at $\tau_{1}$ time scale. Equation 46 is derived versus $\tau_{1}$ time scale:

$$
\nabla_{\left(\boldsymbol{\delta}_{2}, \boldsymbol{N}_{2}, \boldsymbol{\delta}_{1}, \boldsymbol{N}_{1}, \boldsymbol{\delta}_{3}, \boldsymbol{N}_{3}\right)} \widehat{\mathscr{S}}\left(\begin{array}{c}
\frac{\partial \boldsymbol{\delta}_{2}}{\partial \tau_{1}} \\
\frac{\partial \boldsymbol{N}_{2}}{\partial \tau_{1}} \\
\frac{\partial \boldsymbol{\delta}_{1}}{\partial \tau_{1}} \\
\frac{\partial \boldsymbol{N}_{1}}{\partial \tau_{1}} \\
\frac{\partial \boldsymbol{\delta}_{3}}{\partial \tau_{1}} \\
\frac{\partial \boldsymbol{N}_{3}}{\partial \tau_{1}}
\end{array}\right)=\mathbf{0}
$$

Dimension of $\nabla_{\left(\boldsymbol{\delta}_{2}, \boldsymbol{N}_{2}, \boldsymbol{\delta}_{1}, \boldsymbol{N}_{1}, \boldsymbol{\delta}_{3}, \boldsymbol{N}_{3}\right)} \widehat{\mathscr{S}}$ is $2\left(n_{n}+n_{r}\right) \times 2\left(n_{m}+n_{n}+n_{r}\right)$. System of equations 48 can be reorganized as:

$$
\nabla_{\left(\boldsymbol{\delta}_{2}, \boldsymbol{N}_{2}\right)} \widehat{\mathscr{S}}\left(\begin{array}{c}
\frac{\partial \boldsymbol{\delta}_{\mathbf{2}}}{\partial \tau_{1}} \\
\frac{\partial \boldsymbol{N}_{2}}{\partial \tau_{1}}
\end{array}\right)+\nabla_{\left(\boldsymbol{\delta}_{1}, \boldsymbol{N}_{1}, \boldsymbol{\delta}_{3}, \boldsymbol{N}_{3}\right)} \widehat{\mathscr{S}}\left(\begin{array}{c}
\frac{\partial \boldsymbol{\delta}_{1}}{\partial \tau_{1}} \\
\frac{\partial \boldsymbol{N}_{1}}{\partial \tau_{1}} \\
\frac{\partial \boldsymbol{\delta}_{3}}{\partial \tau_{1}} \\
\frac{\partial \boldsymbol{N}_{3}}{\partial \tau_{1}}
\end{array}\right)=\mathbf{0}
$$

Dimension of $\nabla_{\left(\boldsymbol{\delta}_{2}, \boldsymbol{N}_{2}\right)} \widehat{\mathscr{S}}$ is $2\left(n_{n}+n_{r}\right) \times 2 n_{n}$ while dimension of $\nabla_{\left(\boldsymbol{\delta}_{1}, \boldsymbol{N}_{1}, \boldsymbol{\delta}_{3}, \boldsymbol{N}_{3}\right)} \widehat{\mathscr{S}}$ is $2\left(n_{n}+n_{r}\right) \times 2\left(n_{m}+n_{r}\right)$. Equations 46,47 and 49 provide useful tools for detecting equilibrium and singular pints of the system. Equilibrium points of the system can be traced by:

$$
\left\{\begin{array}{c}
\widehat{\mathscr{S}}\left(\boldsymbol{\delta}_{2}, \boldsymbol{N}_{2}, \boldsymbol{\delta}_{1}, \boldsymbol{N}_{1}, \boldsymbol{\delta}_{3}, \boldsymbol{N}_{3}\right)=\mathbf{0} \\
\& \\
\mathbb{M}=0 \\
\& \\
\nabla_{\left(\boldsymbol{\delta}_{2}, \boldsymbol{N}_{2}\right)} \widehat{\mathscr{S}} \text { be invertible }
\end{array}\right.
$$

Singular points of system can be revealed by:

$$
\left\{\begin{array}{c}
\widehat{\mathscr{S}}\left(\boldsymbol{\delta}_{2}, \boldsymbol{N}_{2}, \boldsymbol{\delta}_{1}, \boldsymbol{N}_{1}, \boldsymbol{\delta}_{3}, \boldsymbol{N}_{3}\right)=\mathbf{0} \\
\& \\
\mathbb{M}=0 \\
\& \\
\nabla_{\left(\boldsymbol{\delta}_{2}, \boldsymbol{N}_{2}\right)} \widehat{\mathscr{S}} \text { not to be invertible }
\end{array}\right.
$$


4 Example 1: Time multi scale energy exchanges between a linear oscillator and a coupled nonsmooth NES in the gravitational field

The system under consideration is composed of two coupled oscillators in the gravitational field: the main system is linear with the scalled mass 1 ; it is coupled to a NES with the mass $\epsilon(0<\epsilon \ll 1)$, scalled nonsmooth potential $\epsilon \tilde{F}$ and scalled damping $\epsilon \lambda$. We suppose that the main oscillator is under external scaled force $\epsilon f_{0} \sin (\omega T)$. Governing system equations are summarized as [23]:

$$
\left\{\begin{array}{l}
\ddot{y}_{1}+y_{1}+\epsilon \tilde{F}\left(y_{1}-y_{2}\right)+\epsilon \lambda\left(\dot{y}_{1}-\dot{y}_{2}\right)+\gamma=\epsilon f_{0} \sin (\omega T) \\
\epsilon \ddot{y}_{2}+\epsilon \tilde{F}\left(y_{2}-y_{1}\right)+\epsilon \lambda\left(\dot{y}_{2}-\dot{y}_{1}\right)+\epsilon \gamma=0
\end{array}\right.
$$

with

$$
\tilde{F}(z)= \begin{cases}0 & \text { if }-\delta \leq z \leq \delta \\ k(z-\delta) & \text { if } \quad z \geq \delta \\ k(z+\delta) & \text { if } \quad z \leq-\delta\end{cases}
$$

and $\gamma$ is related to the scaled weight of both oscillators. Since we would like to analyze system behaviors around the $1: 1$ resonance, we set $\omega=1+\sigma \epsilon$.

4.1 Change of variables, complexification and truncated Fourier series of system equations

Since in the system under consideration there is a pre-stressing term $\gamma$, we use the extended version of complex variables of Manevitch (see Eq. 4). By following explained procedures of Sect. 2.1, constant and first terms of the Fourier series read:

$$
\begin{gathered}
\left\{\begin{array}{l}
\frac{1}{1+\epsilon}\left(\frac{b_{1}}{\omega}+\epsilon \frac{b_{2}}{\omega}\right)+(1+\epsilon) \gamma=0 \\
\frac{1}{1+\epsilon}\left(\frac{b_{1}}{\omega}+\epsilon \frac{b_{2}}{\omega}\right)+(1+\epsilon) f_{z}\left(b_{2}, \varphi_{2}, \varphi_{2}^{*}\right)=0
\end{array}\right. \\
\left\{\begin{array}{l}
\dot{\varphi}_{1}=-\frac{i}{2} \epsilon f_{0}+\frac{i}{2 \omega(1+\epsilon)}\left(\varphi_{1}+\epsilon \varphi_{2}\right)-\frac{i}{2} \omega \varphi_{1} \\
\dot{\varphi}_{2}=-\frac{i}{2} \epsilon f_{0}+\frac{i}{2 \omega(1+\epsilon)}\left(\varphi_{1}+\epsilon \varphi_{2}\right)-\frac{\lambda(1+\epsilon)}{2} \varphi_{2}-\frac{i}{2} \omega \varphi_{2}-(1+\epsilon) f_{f}\left(b_{2}, \varphi_{2}, \varphi_{2}^{*}\right)
\end{array}\right.
\end{gathered}
$$

Definitions of $f_{z}\left(b_{2}, \varphi_{2}, \varphi_{2}^{*}\right)$ and $f_{f}\left(b_{2}, \varphi_{2}, \varphi_{2}^{*}\right)$ are given in Appendix (see Eqs. 83-85).

4.2 Time multi scale behavior of the system

For detecting system behaviors at different time scales we implement the explained methods of the Sect. 2.2. 
4.2.1 Constant terms

The general form of the system (54) show that $\gamma=f_{z}\left(N_{2}^{2}\right)$. During the $\epsilon^{0}$ order, it leads to:

$$
b_{1}=-\gamma
$$

while during the $\epsilon^{1}$ order we have:

$$
b_{2}=-\gamma(2+\sigma)
$$

\subsubsection{First harmonics}

Here, we would like to detect time multi-scale responses of first harmonics of the system which are defined in Eq. 55. At fast time scale there is an explicite relation between $\varphi_{1}$ and $\varphi_{2}$ [23]. This means that we should consider part I of the Case 1 in Sect 2.2.1. Equations 12 and 14 read:

$$
\begin{gathered}
\tilde{\Lambda}\left(\varphi_{1}, \varphi_{2}\right)=\frac{i\left(1-G_{f}\left(\left|\varphi_{2}^{2}\right|\right)\right)+\lambda}{2} \varphi_{2}-\frac{i}{2} \varphi_{1}=0 \\
\left(\begin{array}{c}
\delta_{1} \\
N_{1}
\end{array}\right)=h\left(\delta_{2}, N_{2}\right)=\left(\begin{array}{c}
\delta_{2}-\arctan \left(\frac{\lambda}{1-G_{f}\left(N_{2}^{2}\right)}\right) \\
N_{2} \sqrt{\lambda^{2}+\left(1-G_{f}\left(N_{2}^{2}\right)\right)^{2}}
\end{array}\right)
\end{gathered}
$$

Stability borders of the SIM are written as [23]:

$$
\lambda^{2}+\left(1-G_{f}\left(N_{2}^{2}\right)\right)\left(1-2 N_{2} G_{f}^{\prime}\left(N_{2}^{2}\right) N_{2}^{2}-G_{f}\left(N_{2}^{2}\right)\right)>0
$$

At slow time scale, Eq. 18 can be detected by keeping the $\epsilon^{1}$ order of the first equation of the system (55),

$$
\frac{\partial \varphi_{1}}{\partial \tau_{1}}=\mathscr{H}\left(\varphi_{1}, \varphi_{2}\right)=-\frac{i}{2} f_{0}+\frac{i}{2}\left(\varphi_{2}-\varphi_{1}-\sigma \varphi_{1}\right)-\frac{i}{2} \sigma \varphi_{1}
$$

Here, Eq. 19 reads:

$$
g\left(N_{2}\right)\left(\begin{array}{c}
N_{2} \frac{\partial \delta_{2}}{\partial \tau_{1}} \\
\frac{\partial N_{2}}{\partial \tau_{1}}
\end{array}\right)=\widetilde{\mathscr{H}}\left(\delta_{2}, N_{2}\right)=\left(\begin{array}{c}
f_{1}\left(N_{2}, \delta_{2}\right) \\
f_{2}\left(N_{2}, \delta_{2}\right)
\end{array}\right)
$$

$f_{1}\left(N_{2}, \delta_{2}\right), f_{2}\left(N_{2}, \delta_{2}\right)$ and $g\left(N_{2}\right)$ are defined in Appendix (see Eqs. 86-88). Equilibrium points of the system are those which provide $f_{1}\left(N_{2}, \delta_{2}\right)=f_{2}\left(N_{2}, \delta_{2}\right)=0$ and $g\left(N_{2}\right) \neq 0$ while fold singularities are those that give $f_{1}\left(N_{2}, \delta_{2}\right)=f_{2}\left(N_{2}, \delta_{2}\right)=g\left(N_{2}\right)=0$. $g\left(N_{2}\right)=0$ provides two values for $N_{2}$, namely $N_{21}$ and $N_{22}$. They are called as fold lines of the system. Comparing Eqs. 60 and 88, one can see that $g\left(N_{2}\right)=0$ provides stability borders of the SIM. So, fold lines $N_{21}$ and $N_{22}$ are in fact stability borders of the invariant. 
4.3 A numerical example

We set $\delta=1, k=1.5, \lambda=0.2 \gamma=9.81 \times 10^{-4}, \epsilon=10^{-3}, f_{0}=1.6$ and $\sigma=0.1$. Fold line of the system can be obtained via Eq. 88 by setting $g\left(N_{2}\right)=0$ : they read $N_{21}=1.2310$ and $N_{22}=3.2130$. Positions of equilibrium and singular points are presented in Fig. 1 which are obtained by using Eqs. 86-88. It can be seen that for the given forcing amplitude and detuning parameter, the system possesses a pair of fold singularities, namely no.1 and no.2 on the fold line $N_{21}$. There is an equilibrium point which is inside the unstable zone of the system i.e. between fold line $N_{21}$ and $N_{22}$, so it can not be reached. As a summary, the system does not possess any equilibrium points. Existence of fold singularities predicts a SMR for the system under consideration. Let us assume that the initial conditions of Eqs. 52 are $\left(y_{1}(0), y_{2}(0), \dot{y}_{1}(0), \dot{y}_{2}(0)\right)=$

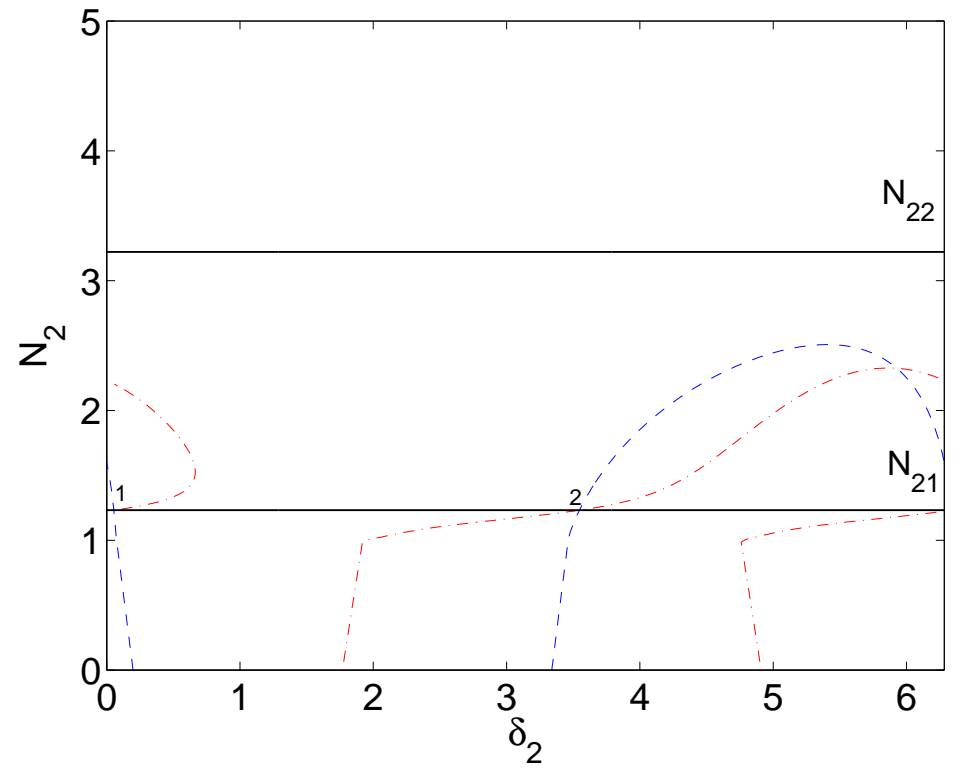

Fig. 1 Positions of equilibrium and singular points for the system under external forcing term $f_{0}=1.6$ and detuning parameter $\sigma=0.1: g\left(N_{2}\right)=0$ or $N_{21}, N_{22}(-), f_{2}\left(N_{2}, \delta_{2}\right)=0(---)$, $f_{1}\left(N_{2}, \delta_{2}\right)=0(-\cdot-\cdot-)$. The zone between $N_{21}$ and $N_{22}$ is unstable. The system possesses a pair of fold singularities on the fold line $N_{21}$, namely no. 1 and no. 2. The system does not possess any equilibrium points.

$\left(1.5+y_{10}, y_{20}, 0,0\right) \cdot y_{10}$ and $y_{20}$ are initial equilibrium positions of the main system and the NES due to their weight (i.e. positions at rest). Equations 52 are directly integrated by the Runge-Kutta method in Matlab via function "ode45". The SIM of the system (Eq. 59), its stability borders (Eq. 60) and included numerical results are depicted in Fig. 2. It is seen that the system presents SMR with repeated bifurcations between its stable zones. This behavior has been already predicted by analytically detection of equilibrium and singular points of the system which have been illustrated in Fig. 1. Histories of amplitudes of two oscillators which are shown in Fig. 3 show the existence of SMR for a quite long time span. 


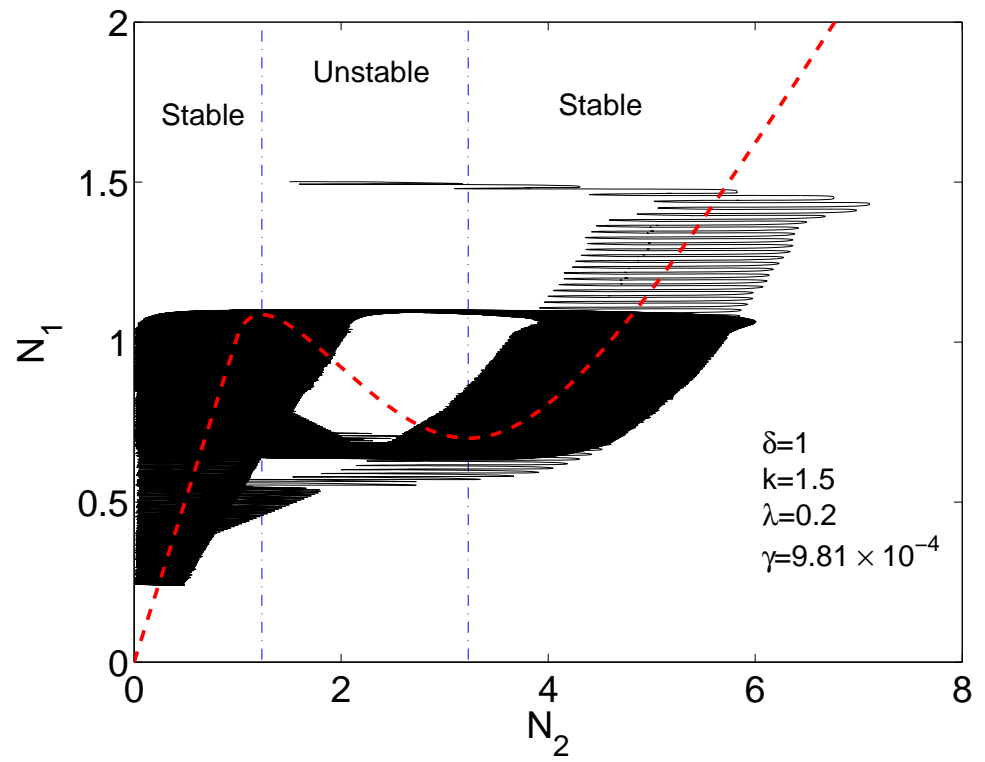

Fig. 2 The SIM $(---)$, its stability borders $(-\cdot-\cdot-)$ and corresponding numerical results $(-)$ of the system with $f_{0}=1.6$ and $\sigma=0.1$. Numerical results are obtained by direct integration of Eqs. (52).

5 Example 2: Time multi-scale energy exchanges between a Bouc-Wen type oscillator coupled to a NES with cubic potential

We consider two coupled oscillators: The main one is with the scaled mass, initial linear stiffness and damping as 1,1 and $\epsilon \zeta$, respectively. It is under external force $\epsilon f_{0} \sin (\omega T)$ and presents a hysteresis behavior of Bouc-Wen type with following carachterstics: the post-yield stiffness $K_{p}$, ratio of the post-yield stiffness and initial stiffnesses as $a$, i.e. $a=\frac{K_{p}}{1}$, and $A, \beta>0, \gamma$ and $n$ are dimensionless parameters parameters which control the hysteresis behavior of the model. This oscillator is coupled to a NES with the scaled mass $\epsilon(0<\epsilon \ll 1)$, scaled damping $\epsilon \lambda$ and the scaled cubic potential function $\epsilon \hat{F}(\alpha)=\epsilon k \alpha^{3}$. If we assume that $z$ and $\dot{z}$ are internal variables for Bouc-Wen model, then governing system equations can be summarized [29]:

$$
\left\{\begin{array}{l}
\ddot{y}_{1}+\epsilon \zeta \dot{y}_{1}+y_{1}+\frac{1-a}{a} z+\epsilon \hat{F}\left(y_{1}-y_{2}\right)+\epsilon \lambda\left(\dot{y}_{1}-\dot{y}_{2}\right)=\epsilon f_{0} \sin (\omega T) \\
\epsilon \ddot{y}_{2}+\epsilon \hat{F}\left(y_{2}-y_{1}\right)+\epsilon \lambda\left(\dot{y}_{2}-\dot{y}_{1}\right)=0 \\
\dot{z}=A \dot{y}_{1}-\beta\left|\dot{y}_{1}\right||z|^{n-1} z-\gamma \dot{y}_{1}|z|^{n}
\end{array}\right.
$$

with $z(0)=0$. We suppose that $k=\mathcal{O}\left(\epsilon^{0}\right)$ and $\frac{1-a}{a}=\epsilon a_{0}=\mathcal{O}\left(\epsilon^{1}\right)$. 
(a)

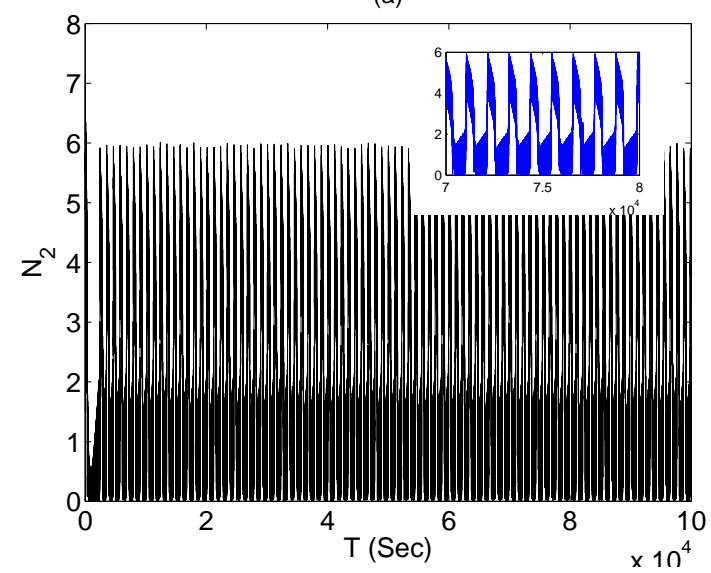

(b)

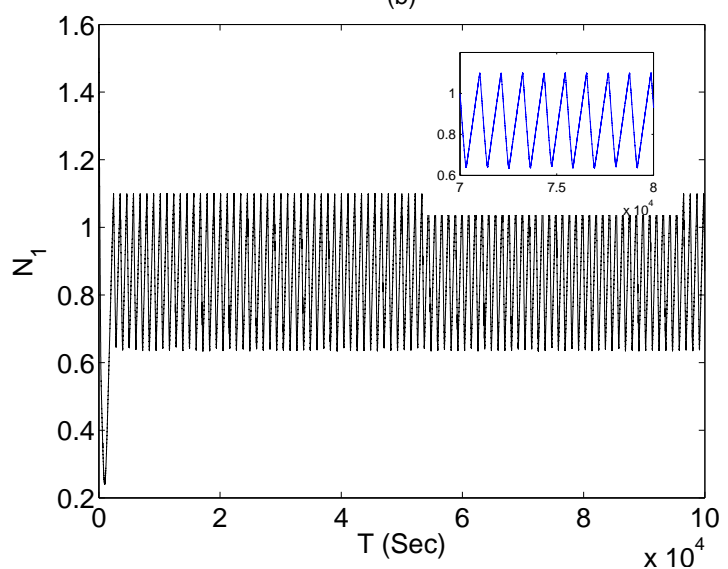

Fig. 3 Histories of system amplitudes with $f_{0}=1.6$ and $\sigma=0.1$ : a) $N_{2}$; b) $N_{1}$. Results are obtained by direct integration of Eqs. (52). Figures are zoomed for the interval of $T \in$ $\left[7 \times 10^{4}, 8 \times 10^{4}\right]$.

5.1 Change of variables, complexification and truncated Fourier series of system equations

Let us follows explained procedure of the Sect. 2.1. In the described system of Eqs. 63 there is not any pre-stressing term. So, in Eq. 4 we set $b_{1}=b_{2}=0$ and we keep just "first harmonics" of the system by setting $l=1$ in Eq. 6 . Following system is obtained 
around the $1: 1$ resonance $(\omega=1+\sigma \epsilon)[29]$ :

$$
\left\{\begin{array}{l}
\dot{\varphi}_{1}+\frac{i}{2}(1+\sigma \epsilon) \varphi_{1}+\frac{\epsilon \zeta}{2(1+\epsilon)}\left(\varphi_{1}+\epsilon \varphi_{2}\right)-\frac{i}{2(1+\epsilon)(1+\sigma \epsilon)}\left(\varphi_{1}+\epsilon \varphi_{2}\right)- \\
\frac{1-a}{a} \frac{i}{2(1+\sigma \epsilon)} \varphi_{3}=\frac{\epsilon f_{0}}{2 i} \\
\dot{\varphi}_{2}+\frac{i}{2}(1+\sigma \epsilon) \varphi_{2}+\frac{\epsilon \zeta}{2(1+\epsilon)}\left(\varphi_{1}+\epsilon \varphi_{2}\right)-\frac{i}{2(1+\epsilon)(1+\sigma \epsilon)}\left(\varphi_{1}+\epsilon \varphi_{2}\right)- \\
\frac{1-a}{a} \frac{i}{2(1+\sigma \epsilon)} \varphi_{3}-\frac{i}{2}(1+\epsilon) \varphi_{2} G\left(\left|\varphi_{2}\right|^{2}\right)+\frac{1+\epsilon}{2} \lambda \varphi_{2}=\frac{\epsilon f_{0}}{2 i} \\
\varphi_{3}=\frac{A}{1+\epsilon}\left(\varphi_{1}+\epsilon \varphi_{2}\right)-\frac{1}{\pi} e^{i \delta_{3}} \frac{\rho}{1+\epsilon}\left(\frac{N_{3}}{\omega}\right)^{n}\left((\beta+\gamma) I\left(n, 0, s_{1}, \text { order of } \epsilon\right)+\right. \\
(-\beta+\gamma) I\left(n, s_{1}, \pi, \text { order of } \epsilon\right)+(-1)^{n}(\beta+\gamma) I\left(n, \pi, s_{2}, \text { order of } \epsilon\right)+ \\
\left.(-1)^{n}(-\beta+\gamma) I\left(n, s_{2}, 2 \pi, \text { order of } \epsilon\right)\right)
\end{array}\right.
$$

with:

$$
\begin{gathered}
G\left(\left|\varphi_{2}\right|^{2}\right)=\frac{3}{4} k\left|\varphi_{2}\right|^{2} \\
\rho=\left(\left(N_{1} \cos \left(\delta_{1}\right)+\epsilon N_{2} \cos \left(\delta_{2}\right)\right)^{2}+\left(N_{1} \sin \left(\delta_{1}\right)+\epsilon N_{2} \sin \left(\delta_{2}\right)\right)^{2}\right)^{\frac{1}{2}} \\
\tan (\theta)=\frac{N_{1} \sin \left(\delta_{1}\right)+\epsilon N_{2} \sin \left(\delta_{2}\right)}{N_{1} \cos \left(\delta_{1}\right)+\epsilon N_{2} \cos \left(\delta_{2}\right)} \\
q(s)=\cos \left(s+\theta-\delta_{3}\right)(\sin (s))^{n} e^{-i s} \\
s_{1}=\frac{\pi}{2}+\delta_{3}-\theta \\
s_{2}=s_{1}+\pi \\
I\left(n, \alpha_{1}, \alpha_{2}, \text { order of } \epsilon\right)=\int_{\alpha_{1}}^{\alpha_{2}} q(s) d s
\end{gathered}
$$

$I\left(n, \alpha_{1}, \alpha_{2}\right.$, order of $\left.\epsilon\right)$ can be calculated for any Bouc-Wen parameter $n$.

5.2 Time multi-scale behavior of the system

At fast time scale the functions $\Lambda$ of Eq. 10 reads:

$$
\Lambda\left(\varphi_{1}, \varphi_{2}, \varphi_{3}\right)=\frac{i\left(1-G\left(\left|\varphi_{2}\right|^{2}\right)\right)+\lambda}{2} \varphi_{2}-\frac{i}{2} \varphi_{1}=0
$$

To deal with all types of Bouc-Wen type behavior with any $n$ parameter, demands a lot of technical computational efforts. So, in this example we focus on the special case 
when $n=3$. The function $\Upsilon$ in Eq. 9 can be obtained from the third equation of the Eq. 64 at $\epsilon^{0}$ order:

$$
\begin{aligned}
& \Upsilon\left(\varphi_{1}, \varphi_{2}, \varphi_{3}\right)=A \varphi_{1}-\frac{1}{\pi} e^{i \delta_{3}} N_{3} N_{1}\left((\beta+\gamma) I\left(3,0, \frac{\pi}{2}+\delta_{3}-\delta_{1}, 0\right)+\right. \\
& (-\beta+\gamma) I\left(3, \frac{\pi}{2}+\delta_{3}-\delta_{1}, \pi, 0\right)+(-1)^{3}(\beta+\gamma) I\left(3, \pi, \frac{3 \pi}{2}+\delta_{3}-\delta_{1}, 0\right)+ \\
& \left.(-1)^{3}(-\beta+\gamma) I\left(3, \frac{3 \pi}{2}+\delta_{3}-\delta_{1}, 2 \pi, 0\right)\right)-\varphi_{3}=0
\end{aligned}
$$

Equation 72 indicates that there is not any explicit relations between $\varphi_{3}, \varphi_{2}$ and $\varphi_{1}$. So, the system under consideration follows Case 2 of the Sect. 2.1. Equation 71 provides following relations:

$$
\begin{aligned}
& \delta_{2}-\arctan \left(\frac{\lambda}{1-G\left(N_{2}^{2}\right)}\right)-\delta_{1}=0 \\
& N_{2} \sqrt{\lambda^{2}+\left(1-G\left(N_{2}^{2}\right)\right)^{2}}-N_{1}=0
\end{aligned}
$$

Equation 74 provides a two-dimentional flow of energies of two oscillators, i.e. $N_{2}$ and $N_{1}$. This SIM has stability borders as [29]:

$$
\lambda^{2}+\left(1-G\left(N_{2}^{2}\right)\right)\left(1-2 N_{2} G^{\prime}\left(N_{2}^{2}\right) N_{2}^{2}-G\left(N_{2}^{2}\right)\right)>0
$$

$G^{\prime}\left(N_{2}^{2}\right)$ is the derivative of $G\left(N_{2}^{2}\right)$ with respect to the $N_{2}^{2}$.

At slow time scale, Eqs. 29 yield to:

$$
\frac{\partial \varphi_{1}}{\partial \tau_{1}}=\mathscr{M}\left(\varphi_{1}, \varphi_{2}, \varphi_{31}, \ldots, \varphi_{3 n}\right)=-\frac{i}{2} f_{0}+\frac{i}{2}(-2 \sigma-1+i \zeta) \varphi_{1}+\frac{i}{2} \varphi_{2}+\frac{i a_{0}}{2} \varphi_{3}
$$

After consideration of definitions of $\varphi_{1}$ and $\varphi_{3}$ from Eqs. 71 and 72 in Eq. 76, Eq. 34 reads [29]:

$$
g_{1}\left(N_{2}\right)\left(\begin{array}{c}
N_{2} \frac{\partial \delta_{2}}{\partial \tau_{1}} \\
\frac{\partial N_{2}}{\partial \tau_{1}}
\end{array}\right)=\left(\begin{array}{c}
\digamma_{1}\left(N_{2}, \delta_{2}\right) \\
\digamma_{2}\left(N_{2}, \delta_{2}\right)
\end{array}\right)=\left(\begin{array}{c}
\lambda \Xi+\left(1-G\left(N_{2}^{2}\right)-2 N_{2}^{2} G^{\prime}\left(N_{2}^{2}\right)\right) \Theta \\
\left(1-G\left(N_{2}^{2}\right)\right) \Xi-\lambda \Theta
\end{array}\right)
$$

$\Xi, \Theta$ and $g_{1}\left(N_{2}\right)$ are presented in Appendix (see Eqs. 89-91). Eq. 91 is also stability borders of the SIM of the system which already defined in Eq.75. Equilibrium and singular points of the system can be traced via 77: Equilibrium points are those that provide $\digamma_{1}=\digamma_{2}=0$ and $g_{1}\left(N_{2}\right) \neq 0$. Singular points of the system are those which give $\digamma_{1}=\digamma_{2}=g_{1}\left(N_{2}\right)=0$. Let us set $\digamma_{1}=\digamma_{2}=0$. We will have:

$$
\left\{\begin{array}{l}
\left(1-G\left(N_{2}^{2}\right)\right) \Xi-\lambda \Theta=0 \\
\lambda \Xi+\left(1-G\left(N_{2}^{2}\right)-2 N_{2}^{2} G^{\prime}\left(N_{2}^{2}\right)\right) \Theta=0
\end{array}\right.
$$

Equation 78 is a homogeneous system of two equations with respect to $\Xi$ and $\Theta$. Its characteristic equation which provides its eigenvalues is equal to $g_{1}\left(N_{2}\right)=0$. If 
$g_{1}\left(N_{2}\right) \neq 0$, then $\Xi=\Theta=0$ will be only solutions of the homogeneous system of two equations. So, the system at equilibrium points reads:

$$
\left\{\begin{array}{l}
-\frac{1}{2} f_{0} \sin \left(\delta_{2}\right)-\frac{1}{2} N_{2} \lambda(2 \sigma+1)-\frac{1}{2} N_{2} \zeta\left(1-G\left(N_{2}^{2}\right)\right)- \\
\frac{1}{2} N_{3} a_{0} \sin \left(\delta_{3}-\delta_{2}\right)=0 \\
-\frac{1}{2} f_{0} \cos \left(\delta_{2}\right)-\frac{1}{2} N_{2}\left(1-G\left(N_{2}^{2}\right)\right)(2 \sigma+1)+\frac{1}{2} N_{2} \zeta \lambda+\frac{1}{2} N_{2}+ \\
\frac{1}{2} N_{3} a_{0} \cos \left(\delta_{3}-\delta_{2}\right)=0
\end{array}\right.
$$

From Eq. 79 following relations can be obtained:

$$
\begin{aligned}
& N_{3}=\frac{1}{a_{0}}\left(\left(-f_{0} \sin \left(\delta_{2}\right)-N_{2} \lambda(2 \sigma+1)-N_{2} \zeta(1-G)\right)^{2}+\right. \\
& \left.\left(-f_{0} \cos \left(\delta_{2}\right)-N_{2}(1-G)(2 \sigma+1)+N_{2} \zeta \lambda+N_{2}\right)^{2}\right)^{\frac{1}{2}} \\
& \delta_{3}=\delta_{2}-\sin ^{-1}\left(\frac{f_{0} \sin \left(\delta_{2}\right)+N_{2} \lambda(2 \sigma+1)+N_{2} \zeta(1-G)}{a_{0} N_{3}}\right)
\end{aligned}
$$

Let us define real and imaginary parts of the Eq.(72), as $C_{1}$ and $C_{2}$, respectively. Equilibrium points of the system in the surface of $\left(\delta_{2}, N_{2}\right)$ can be traced via consideration of the definition of $N_{3}$ and $\delta_{3}$ from Eqs. 80 and 81 in $C_{1}\left(\delta_{2}, N_{2}\right)=C_{2}\left(\delta_{2}, N_{2}\right)=0$. Amplitudes of fold singularities of the system can be detected by setting $g_{1}\left(N_{2}\right)=0$ which provides two values for $N_{2}$ (fold lines), namely $N_{21}$ and $N_{22}$ :

$$
\begin{aligned}
& N_{21}=\frac{2}{3} \sqrt{\frac{2}{k}-\frac{\sqrt{1-3 \lambda^{2}}}{k}} \\
& N_{22}=\frac{2}{3} \sqrt{\frac{2}{k}+\frac{\sqrt{1-3 \lambda^{2}}}{k}}
\end{aligned}
$$

Phases in the fold lines are obtained by injecting Eqs. 80 and 82 in $C_{1}\left(\delta_{2}, \delta_{3}\right)=$ $C_{2}\left(\delta_{2}, \delta_{3}\right)=0$.

\subsection{A numerical example}

Let us assume following system parameters: $\epsilon=10^{-3}, \lambda=0.2, \zeta=0.1, \sigma=1$, $A=1, a=0.99, \beta=0.5, \gamma=0, n=3, k=0.01$ and $f_{0}=15$. We assume that $y_{1}(0)=10$ and $y_{2}(0)=\dot{y}_{1}(0)=\dot{y}_{2}(0)=0$. Equations 63 are integrated directly by "ode45" function of Matlab and are compared with analytical predictions. Positions of equilibrium points are highlighted in Fig. 4. The system possesses two equilibrium points, namely no. 1 and no. 2. There are other equilibrium points but their positions are in the unstable zone of the system, i.e. between two fold line, so they are unstable and we do not consider them. Singular points should be detected on fold lines $N_{21}$ and $N_{22}$. Their locations on these lines are illustrated in Figs. 5 and 6 , respectively. As a summary there is a pair of singular points on the fold line $N_{21}$ while fold line $N_{22}$ does not host any singular points. The SIM of the system obtained from Eq. 74 and 
corresponding results from numerical integration of the Eqs. 63 are depicted in Fig. 7. Histories of system amplitudes detected via numerical integration are shown in Fig. 8. These figures show that the system presents SMR which houses repeated bifurcations between its stability borders. This behavior is due to existence of singular points on the first fold line which prepare the system for reverse bifurcations. The energy of the main oscillator is trapped in the closed loop cycle. This behavior is desirable from passive control view point if the system behavior during the closed loop bifurcations via SMR, presents allowable energy variations for the main oscillator. This can be carried out by appropriate design of the NES. The system possesses two equilibrium points (see Fig. 4) which can be stable or unstable; they can be seen after very long time during $\tau_{1}$ time scale or during other time scales such as $\tau_{2}$ time scale. Detection of this behavior via numerical integration of main equations demands a lot of computational efforts and is out of the scope of the current paper.

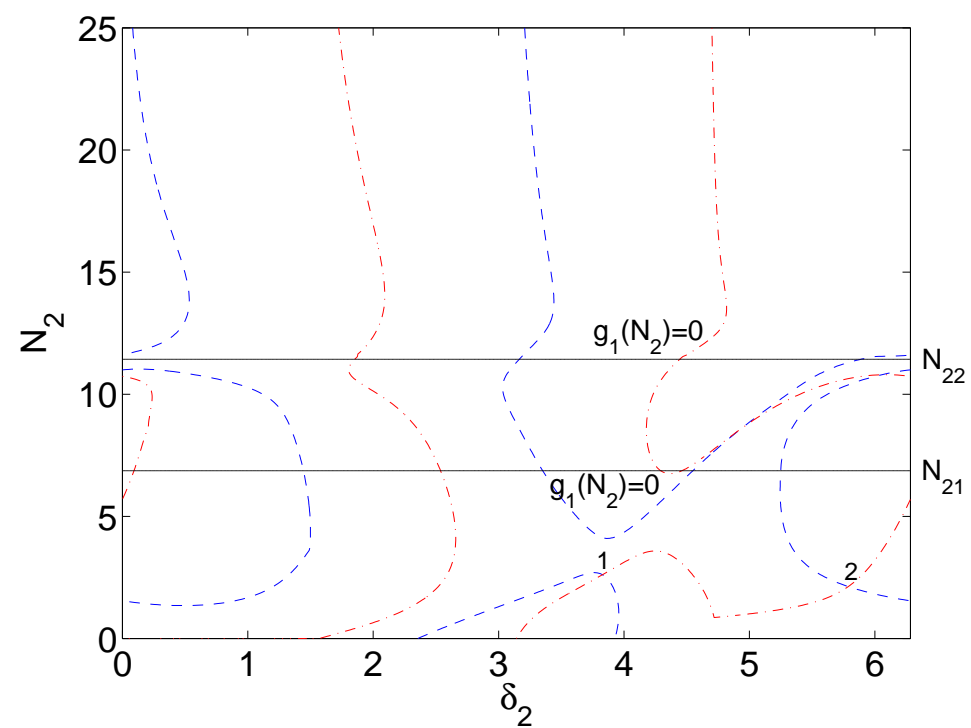

Fig. 4 Positions of equilibrium points for the system under external forcing term $f_{0}=15$ : $g_{1}\left(N_{2}\right)=0(-)$, i.e. $N_{21}, N_{22}$ lines, $C_{1}\left(N_{2}, \delta_{2}\right)=0(---), C_{2}\left(N_{2}, \delta_{2}\right)=0,(-\cdot-\cdot-)$ : the system has two equilibrium points namely no. 1 and no. 2 .

\section{Conclusions}

A general methodology for detection of time multi-scale behaviors of general coupled oscillators is presented. The first set of the oscillators is considered to be the main ones and the other set is nonlinear energy sink devices with very light masses compared to the masses of the first set. The rheology of the system can be represented via constitutive laws by either differential inclusions, differential equations or algebraic relations between internal variables. Step by step treatments of primary system equations in the complex domain permit us to use a Galerkin technique by keeping constant terms and 


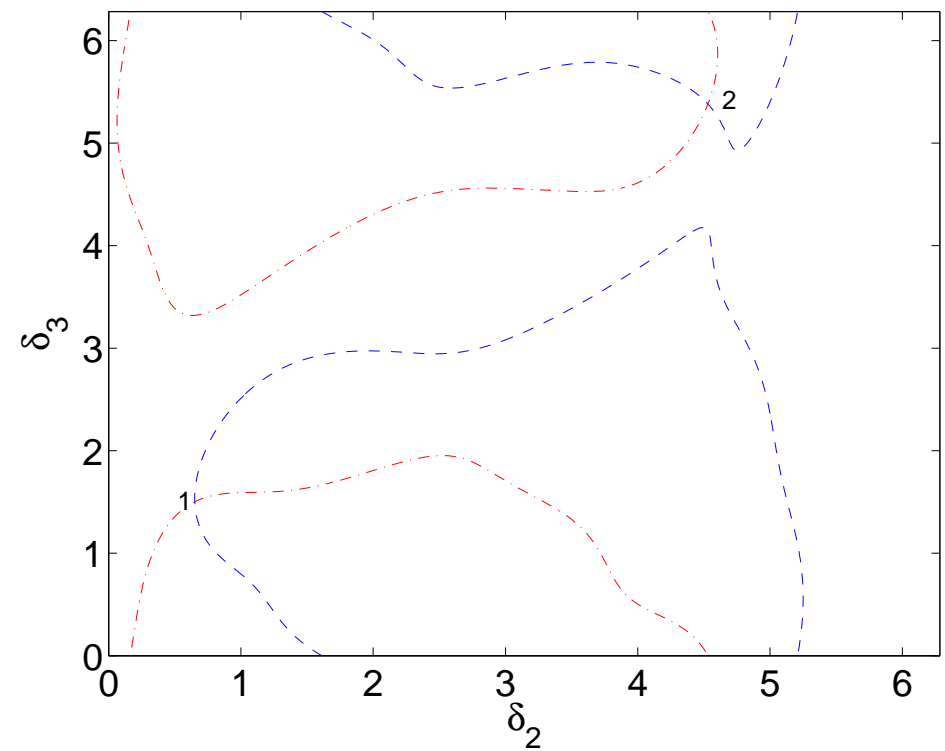

Fig. 5 Positions of fold singularities for the system under external forcing term $f_{0}=15$ on the fold line $N_{21}: C_{1}\left(\delta_{2}, \delta_{3}\right)=0(---), C_{2}\left(\delta_{2}, \delta_{3}\right)=0,(-\cdot-\cdot-)$. The system has two singular points namely no.1 and no. 2 , on the fold line $N_{21}$.

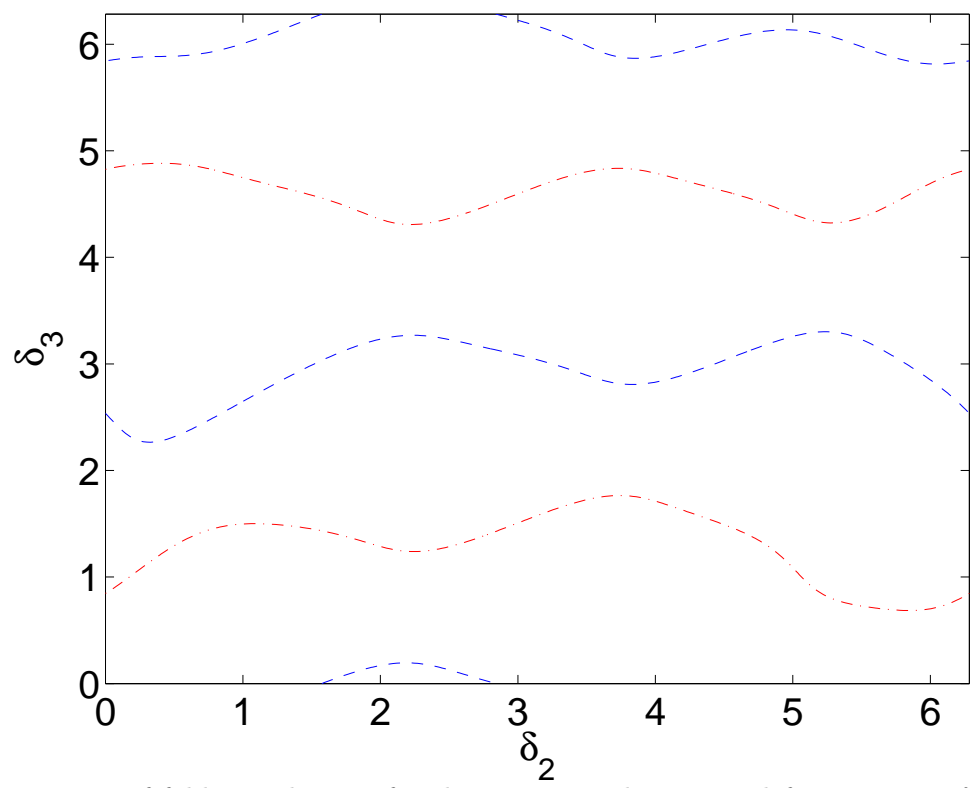

Fig. 6 Positions of fold singularities for the system under external forcing term $f_{0}=15$ on the fold line $N_{22}: C_{1}\left(\delta_{2}, \delta_{3}\right)=0(---), C_{2}\left(\delta_{2}, \delta_{3}\right)=0,(-\cdot-\cdot-)$. The system does not have any singular point on the fold line $N_{22}$. 


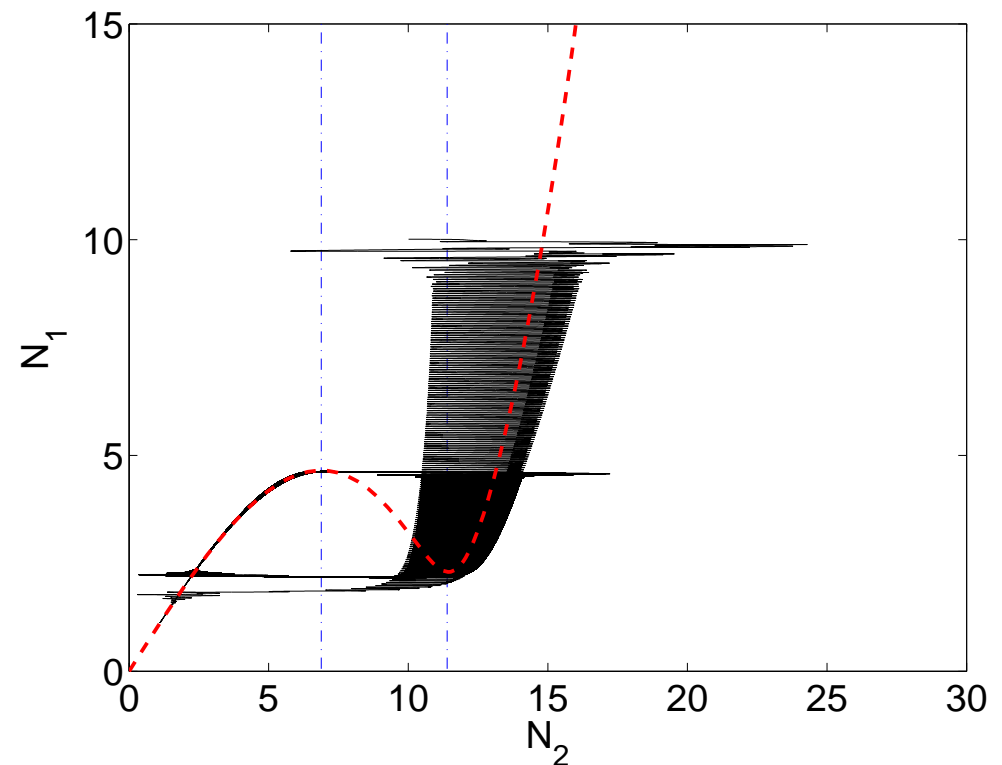

Fig. 7 The SIM $(---)$, its stability borders $(-\cdot-\cdot-)$ and corresponding numerical results $(-)$ for the system with $f_{0}=15$. Numerical results are obtained via direct integration of Eqs. 63.

first harmonics of the Fourier series. Studying the system at fast time scale provides its invariant while its behavior at slow time scale provides positions of equilibrium and singular points on the invariant. Equilibrium and singular points correspond to periodic and possible strongly modulated responses, respectively. Application of the proposed methodology is demonstrated in two special cases representing two different coupled oscillators with different rheologies. Developed analytical tools permit us to design "desired" equilibrium and singular points which is equivalent to the design of nonlinear energy sink according to the demand, which can be passive control and/or energy harvesting of main oscillators.

Acknowledgements The authors would like to thank following organizations for supporting this research: i) The "Ministère de l'Écologie, du Développement Durable et de l'Énergie" and ii) LABEX CELYA (ANR-10-LABX-0060) of the "Universite de Lyon" within the program "Investissement d'Avenir" (ANR-11-IDEX-0007) operated by the French National Research Agency (ANR). 


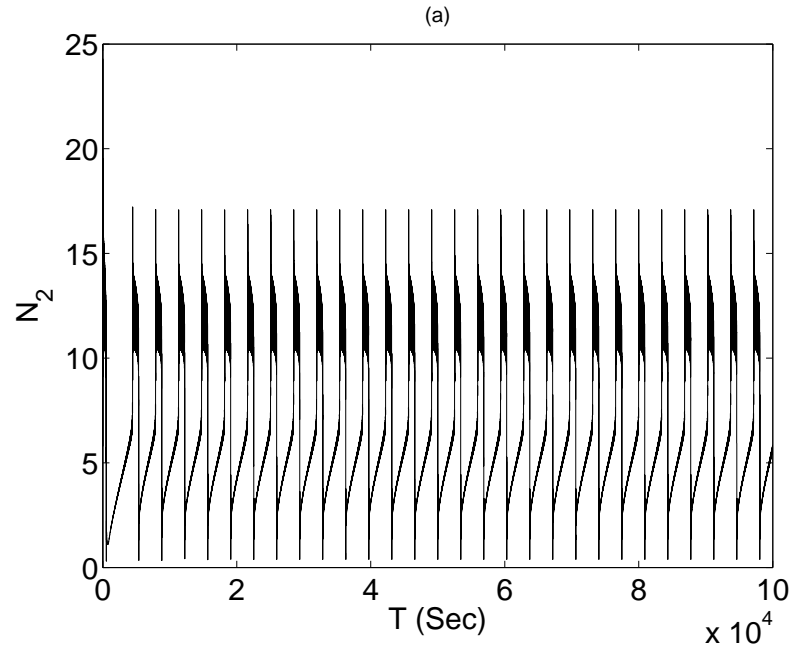

(b)

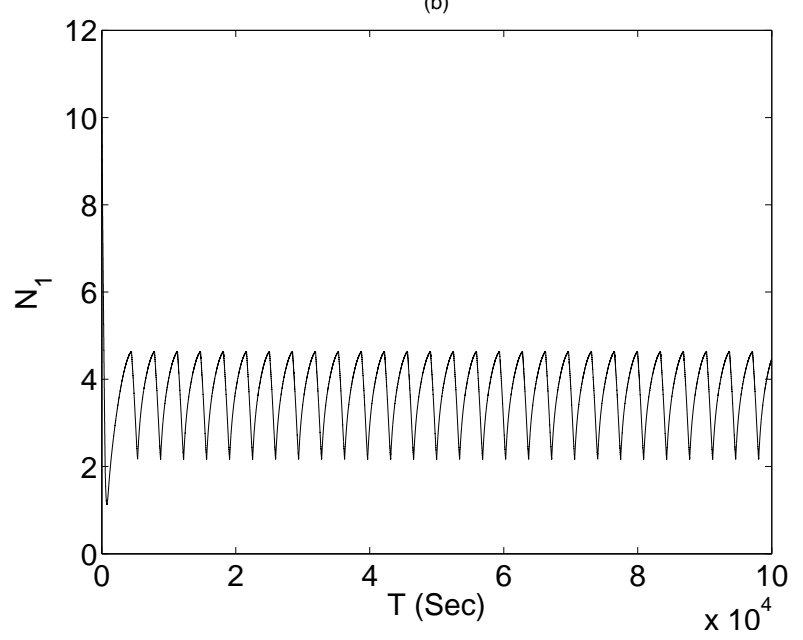

Fig. 8 Histories of system amplitudes with $f_{0}=15$ : a) $N_{2}$; b) $N_{1}$. These results are obtained via direct integration of Eqs. 63.

\section{Appendix}

For any variable $\chi \geq 0$

$$
f_{z}(\chi)=\left\{\begin{array}{l}
0 \quad \text { if } \frac{b_{2}}{\omega}+\frac{\sqrt{\chi}}{\omega}<\delta \\
\frac{k}{\pi \omega}\left(b_{2} \pi+\sqrt{\left(b_{2}+\sqrt{\chi}-\delta \omega\right)(-b+\sqrt{\chi}+\delta \omega)}-\right. \\
\sqrt{\left(-b_{2}+\sqrt{\chi}-\delta \omega\right)\left(b_{2}+\sqrt{\chi}+\delta \omega\right)}+ \\
\left.\left(b_{2}-\delta \omega\right) \arcsin \left(\frac{b_{2}-\delta \omega}{\sqrt{\chi}}\right)+\left(b_{2}+\delta \omega\right) \arcsin \left(\frac{b_{2}+\delta \omega}{\sqrt{\chi}}\right)\right) \\
\quad \text { if } \frac{b_{2}}{\omega}+\frac{\sqrt{\chi}}{\omega} \geq \delta
\end{array}\right.
$$




$$
\begin{aligned}
& f_{f}\left(b_{2}, \varphi_{2}, \varphi_{2}^{*}\right)=-\frac{i \varphi_{2}}{2} G_{f}\left(\left|\varphi_{2}\right|^{2}\right) \\
& G_{f}(\chi)=\left\{\begin{array}{l}
0 \quad \text { if } \frac{b_{2}}{\omega}+\frac{\sqrt{\chi}}{\omega}<\delta \\
\frac{k}{2 \pi \omega}\left(\pi+\frac{2 b_{2}}{\chi} \sqrt{\chi-\left(b_{2}-\delta \omega\right)^{2}}-\frac{2 \delta \omega}{\chi} \sqrt{\chi-\left(b_{2}-\delta \omega\right)^{2}}-\right. \\
\frac{2 b_{2}}{\chi} \sqrt{\chi-\left(b_{2}+\delta \omega\right)^{2}}-\frac{2 \delta \omega}{\chi} \sqrt{\chi-\left(b_{2}+\delta \omega\right)^{2}}+ \\
\left.2 \arccos \left(\frac{b_{2}+\delta \omega}{\sqrt{\chi}}\right)+2 \arcsin \left(\frac{b_{2}-\delta \omega}{\sqrt{\chi}}\right)\right) \quad \text { if } \frac{b_{2}}{\omega}+\frac{\sqrt{\chi}}{\omega} \geq \delta
\end{array}\right. \\
& f_{1}\left(N_{2}, \delta_{2}\right)=-\left(1-G_{f}\left(N_{2}^{2}\right)-2 N_{2}^{2} G_{f}^{\prime}\left(N_{2}^{2}\right)\right) f_{0} \cos \left(\delta_{2}\right)-\lambda f_{0} \sin \left(\delta_{2}\right)- \\
& N_{2} \lambda^{2}(1+2 \sigma)+N_{2}\left(1-G_{f}\left(N_{2}^{2}\right)-2 N_{2}^{2} G_{f}^{\prime}\left(N_{2}^{2}\right)\right)\left(-2 \sigma+2 \sigma G_{f}\left(N_{2}^{2}\right)+G_{f}\left(N_{2}^{2}\right)\right) \\
& f_{2}\left(N_{2}, \delta_{2}\right)=f_{0} \sin \left(\delta_{2}\right)\left(G_{f}\left(N_{2}^{2}\right)-1\right)-\lambda N_{2}+\lambda f_{0} \cos \left(\delta_{2}\right) \\
& \left.g\left(N_{2}\right)=2\left(\lambda^{2}+\left(1-G_{f}\left(N_{2}^{2}\right)\right)\left(1-2 N_{2} G_{f}^{\prime}\left(N_{2}^{2}\right) N_{2}^{2}-G_{f}\left(N_{2}^{2}\right)\right)\right)\right) \\
& \Xi=-\frac{1}{2} f_{0} \sin \left(\delta_{2}\right)-\frac{1}{2} N_{2} \lambda(2 \sigma+1)-\frac{1}{2} N_{2} \zeta\left(1-G\left(N_{2}^{2}\right)\right)- \\
& \frac{1}{2} N_{3} a_{0} \sin \left(\delta_{3}-\delta_{2}\right) \\
& \Theta=-\frac{1}{2} f_{0} \cos \left(\delta_{2}\right)-\frac{1}{2} N_{2}\left(1-G\left(N_{2}^{2}\right)\right)(2 \sigma+1)+\frac{1}{2} N_{2} \zeta \lambda+\frac{1}{2} N_{2}+ \\
& \frac{1}{2} N_{3} a_{0} \cos \left(\delta_{3}-\delta_{2}\right) \\
& g_{1}\left(N_{2}\right)=\left(-1+G\left(N_{2}^{2}\right)\right)^{2}+\lambda^{2}+2\left(-1+G\left(N_{2}^{2}\right)\right) N_{2}^{2} G^{\prime}\left(N_{2}^{2}\right)
\end{aligned}
$$

\section{References}

1. Frahm, H.: Device for damping vibrations of bodies, Apr. 18 1911. US Patent 989,958.

2. Gendelman, O., Manevitch, L. I., Vakakis, A. F., MCloskey, R.: Energy pumping in nonlinear mechanical oscillators: part $\mathrm{i}$ - dynamics of the underlying hamiltonian systems. Journal of Applied Mechanics, 68 (1), 34-41 (2001).

3. Vakakis A. F.: Inducing passive nonlinear energy sinks in vibrating systems. Journal of Vibration and Acoustics, 123(3), 324-332 (2001).

4. Kerschen, G., Vakakis, A. F., Lee, Y. S., McFarland, D. M., Kowtko, J. J., Bergman, L. A.: Energy transfers in a system of two coupled oscillators with essential nonlinearity: 1:1 resonance manifold and transient bridging orbits. Nonlinear Dynamics, 42, 283-303 (2005).

5. Gourdon, E., Lamarque, C.-H.: Energy pumping for a larger span of energy. Journal of Sound and Vibration 285, 711-720 (2005).

6. Kerschen, G., Lee, Y. S., Vakakis, A. F., McFarland, D. M., Bergman, L. A.: Irreversible passive energy transfer in coupled oscillators with essential nonlinearity. SIAM Journal of Applied Mathematics, 66, 648-679, (2006)

7. Manevitch, L. I., Gourdon, E., Lamarque, C.-H.: Parameters optimization for energy pumping in strongly nonhomogeneous 2 dof system. Chaos Soliton \& Fracal, 31, 900-911 (2007). 
8. Starosvetsky, Y, Gendelman, O. V.: Vibration absorption in systems with a nonlinear energy sink: nonlinear damping. Journal of Sound and Vibration, 324, 916-939 (2009)

9. Gendelman, O. V., Vakakis, A. F., Bergman, L. A., McFarland, D. M.: Asymptotic analysis of passive nonlinear suppression of aeroelastic instabilities of a rigid wing in subsonic flow. SIAM Journal of Applied Mathematics, 70, 1655-1677 (2010).

10. Vaurigaud, B., Ture Savadkoohi, A., Lamarque, C.-H.: Targeted energy transfer with parallel nonlinear energy sinks Part I: Design theory and numerical results. Nonlinear Dynamics, 66 (4), 763-780 (2011).

11. Luongo, A., Zulli, D.: Dynamic analysis of externally excited NES-controlled systems via a mixed Multiple Scale/Harmonic Balance algorithm. Nonlinear Dynamics, 70, 2049-2061 (2012)

12. Ture Savadkoohi, A., Vaurigaud, B., Lamarque C.-H., Pernot, S.: Targeted energy transfer with parallel nonlinear energy sinks, part II: theory and experiments. Nonlinear Dynamics, 67, 37-46 (2012)

13. Wierschem, N. E., Quinn, D. D., Hubbard, S. A., Al-Shudeifatd, M. A., McFarlandc, D. M., Luo, J., Fahnestock, L. A., Spencer Jr, B. F., Vakakis, A. F., Bergman, L. A.: Passive damping enhancement of a two-degree-of-freedom system through a strongly nonlinear twodegree-of-freedom attachment. Journal of Sound and Vibration, 331, 5393-5407 (2012)

14. Luongo, A., Zulli, D.: Aeroelastic instability analysis of NES-controlled systems via a mixed Multiple Scale/Harmonic Balance Method. Journal of Vibration and Control, 20, 1985-1998 (2014)

15. Vakakis, A. F., Gendelman, O. V., Bergman, L. A., McFarland, D. M., Kerschen, G., Lee, Y. S.: Nonlinear trageted energy transfer in mechanical and structural systems I \& II. Springer, Berlin (2008)

16. Vaurigaud, B., Manevitch, L. I., Lamarque, C.-H.: Passive control of aeroelastic instability in a long span bridge model prone to coupled flutter using targeted energy transfer. Journal of Sound and Vibration, 330 (11), 2580-2595 (2011)

17. Nucera, F., Vakakis, A. F., McFarland, D. M., Bergman, L. A., Kerschen, G.: Targeted energy transfers in vibro-impact oscillators for seismic mitigation. Nonlinear Dynamics, 50, 651-677 (2007)

18. Gendelman, O. V.: Targeted energy transfer in systems with non-polynomial nonlinearity. Journal of Sound and Vibration, 315, 732-745 (2008).

19. Gendelman, O. V.: Analytic treatment of a system with a vibro-impact nonlinear energy sink. Journal of Sound and Vibration, 331, 4599-4608 (2012).

20. Gourc, E., Michon, M., Seguy, S., Berlioz, A.: Targeted energy transfer under harmonic forcing with a vibro-impact nonlinear energy sink: analytical and experimental developments. Journal of Vibration and Acoustics, 137, 031008 (2015)

21. Gendelman, O. V., Alloni, A.: Dynamics of forced system with vibro-impact energy sink. Journal of Sound and Vibration, 358, 301-314 (2015)

22. Lamarque, C.-H., Gendelman, O. V., Ture Savadkoohi, A., Etcheverria, E.: Targeted energy transfer in mechanical systems by means of non-smooth nonlinear energy sink. Acta Mechanica. 221, 175-200 (2011)

23. Ture Savadkoohi, A., Lamarque, C.-H., Dimitrijevic, Z.: Vibratory energy exchange between a linear and a non-smooth system in the presence of the gravity. Nonlinear Dynamics, 70, 1473-1483 (2012)

24. Lamarque, C.-H., Ture Savadkoohi, A., Dimitrijevic, Z.: Dynamics of a linear system with time-dependant mass and a coupled light mass with non-smooth potential. Meccanica, 49, $135-145$ (2014)

25. Ture Savadkoohi, A., Lamarque, C.-H.: Vibratory energy localization by non-smooth energy sink with time-varying mass. Applied Non-Linear Dynamical Systems. Springer Proceedings in Mathematics \& Statistics. 93, 429-442 (2014)

26. Lamarque, C.-H., Ture Savadkoohi, A., Etcheverria, E., Dimitrijevic, Z.: Multi-scales dynamics of two coupled non-smooth systems. International Journal of Bifurcation and Chaos, 22, 1250295:1-18 (2012).

27. Ture Savadkoohi, A., Lamarque, C.-H.: Dynamics of coupled Dahl type and non-smooth systems at different scales of time. International Journal of Bifurcation and Chaos, 23, 1350114: 1-14 (2013).

28. Weiss, M., Chenia, M., Ture Savadkoohi, A., Lamarque, C.-H., Vaurigaud, B., Hammouda, A.: Multi-scale energy exchanges between an elasto-plastic oscillator and a light nonsmooth system with external pre-stress. Nonlinear Dynamics, 83, 109-135 (2016) 
29. Lamarque, C.-H., Ture Savadkoohi, A.: Dynamical behavior of a Bouc-Wen type oscillator coupled to a nonlinear energy sink. Meccanica, 49, 1917-1928 (2014)

30. Bastien, J., Bernardin, F., Lamarque, C.-H.: Non Smooth Deterministic or Stochastic Discrete Dynamical Systems: Applications to Models with Friction or Impact. Wiley, 515 (2013).

31. Weiss, M., Ture Savadkoohi, A., Gendelman, O. V., Lamarque, C.-H.: Dynamical behavior of a mechanical system including saint-venant component coupled to a nonlinear energy sink. Internation Journal of Non-Linear Mechanics, 63, 10-18 (2014)

32. Lamarque, C.-H., Ture Savadkoohi, A.: Targeted energy transfer between a system with a set of Saint-Venant elements and a nonlinear energy sink. Continuum Mechanics and Thermodynamics, 27 (4), 819-833 (2015).

33. Manevitch L. I., The description of localized normal modes in a chain of nonlinear coupled oscillators using complex variables. Nonlinear Dynamics, 25, 95-109 (2001).

34. Starosvetsky Y, Gendelman O. V., Strongly modulated response in forced 2DOF oscillatory system with essential mass and potential asymmetry. Physica D, 237, 1719-1733 (2008).

35. Ikhouane F. Rodellar J., Systems with Hysteresis. Wiley, West Sussex (2007).

36. Nayfeh A. H., Mook D. T., Nonlinear oscillations, 720, John Wiley and Sons, New York (1979). 